\title{
Two-component Fermi systems. I. Fluid coupled cluster theory
}

\author{
R F Bishop and W A Lahoz $†$ \\ Department of Mathematics, University of Manchester Institute of Science and Technology, \\ PO Box 88, Manchester M60 1QD, UK
}

Received 5 January 1987, in final form 9 March 1987

\begin{abstract}
This paper is the first of two in which the coupled cluster method (CCM) or $\exp (S)$ formalism is applied to two-component Fermi systems, the aim being to describe real metals and superconductivity. In this paper we concentrate on exact results and restrict ourselves to a ring approximation, applicable essentially to a high-density regime. We show that in the ground-state formalism the random phase approximation (RPA) can be formulated as a system of coupled, bilinear integral equations satisfied by functions associated with the so-called four-point functions of the system which provide a measure of the two-particle-two-hole component in the true ground-state wavefunction. These equations are analysed in the dimensionless parameter formed by the ratio of the species masses and exact analytic solutions obtained. For Coulombic potentials $\left(V_{11} V_{22}=V_{12}^{2}\right)$ we show that the exact analytic solution is unique and obtain an expression for the correlation energy. For non-Coulombic potentials $\left(V_{11} V_{22} \neq V_{12}^{2}\right)$ we indicate how to obtain a possible analytic solution. An RPA-like treatment of the one- and two-body equations in the excited-state formalism is provided for completeness.
\end{abstract}

\section{Introduction}

Two-component systems involving two types of particles, namely ' 1 ' and '2', interacting via the repulsive potentials $V_{11}$ and $V_{22}$ and the attractive potential $V_{12}$, all assumed to possess a Fourier transform, are natural generalisations of one-component systems. They provide a framework within which to study systems such as real metals and electron-hole plasmas in photoexcited semiconductors, and phenomena such as Bardeen-Cooper-Schrieffer (BCS) superconductivity (Bardeen et al 1957, Bogoliubov 1958, Valatin 1958).

A measure of the density of a degenerate ensemble of identical charged particles is provided by the dimensionless parameter $r_{\mathrm{s}}$ (the smallness parameter in a perturbation-theoretic treatment), defined as the ratio of the average interparticle spacing $r_{0}$ to the Bohr radius $a_{0}$. Exact calculations have been performed in the high density $\left(r_{\mathrm{s}} \rightarrow 0\right)$ (Gell-Mann and Brueckner 1957) and low density $\left(r_{\mathrm{s}} \rightarrow \infty\right)$ (Wigner 1934, 1938) limits, the aim being to interpolate between them to describe real metals which have densities $2 \leqslant r_{s} \leqslant 6$. The early calculations with this goal in mind were mainly perturbational or variational in character (cf Bishop and Lürhmann 1978, Lahoz 1986 for a review). Recently, the coupled cluster method ( $\mathrm{CCM}$ ) has been applied to this problem with great success in obtaining both analytic and numerical (Bishop and Lührmann 1978, 1982, Emrich and Zabolitzky 1984) results of great quality. This suggests that real

$\dagger$ Present address: Department of Meteorology, University of Edinburgh, James Clerk Maxwell Building, King's Buildings, Mayfield Road, Edinburgh EH9 3JZ, UK. 
metals, made up of electrons and ions, may be accurately described by a two-component CCM formalism.

It is well known that the effective attractive interaction between electrons just inside the Fermi surface sufficient for a non-zero gap, i.e. superconducting solutions in BCS theory (cf Fetter and Walecka 1971), can be brought about by the intervention of a different type of particle (Cooper 1956). In the standard one-component treatment (Bogoliubov 1958, Valatin 1958) an effective attractive interaction, duly accounted for, is assumed from the beginning and the well known results obtained. The nature of BCS superconductivity, however, suggests that a two-component treatment will provide a better understanding of this phenomenon.

The CCM, based on the $\exp (S)$ ansatz of Hubbard (1957), was originated by Coester and Kümmel (1960, Coester 1958, 1969). In the so-called fluid ground state the normal CCM (to differentiate it from the extended CCM of Arponen (1983)) has been applied with great success to a wide variety of many-body problems including: (i) closed-shell atomic nuclei (Zabolitzky 1974a, b, Kümmel et al 1978); (ii) the electron gas (Bishop and Lührmann 1978, 1982, Emrich and Zabolitzky 1984); and (iii) examples from quantum chemistry (Čižek 1966, 1969, Lindgren 1978, Kvasnička et al 1982, Szalewicz et al 1984).

The extension to excited states has been via two formalisms. The first is due to Offermann, Kümmel and Ey (OKE), and was developed to deal with open-shell nuclear systems. It now provides a direct microscopic foundation to the very successful phenomenological shell-model calculations for the excited states of atomic nuclei (Offermann et al 1976, Offermann 1975, 1976). The second approach is due to Emrich, and it is this which we henceforth refer to as the fluid excited-state formalism. It can be regarded as the natural extension of the fluid ground state to the excited states, at least for homogeneous systems (Emrich 1981a, b), and it is a priori expected to prove as successful as its fluid ground-state counterpart.

Of great importance has been the development by Arponen (1983) of an $\exp (S)$ formalism derived from a variational principle and seemingly a generalisation of the normal CCM. This formalism allows one to obtain the expectation values of arbitrary operators and appears capable of handling phase transitions in simple models, e.g. LMG (Lipkin et al 1965a, b, c); however, it has not yet proved capable of handling more complicated systems such as liquid ${ }^{4} \mathrm{He}$, although work is in progress (Robinson 1986).

In the fermionic fluid ground-state formalism one generally chooses a single Slater determinant of occupied states as a model state and makes the usual $\exp (S)$ ansatz for the true ground-state wavefunction, $S$ being an operator containing 1-body, 2 body, $\ldots, N$-body pieces. It can be shown (Bishop and Kümmel 1987) that the wavefunction obtained by correlating all possible $n$-tuplets from the filled Fermi sea is given precisely by the $\exp (S)$ ansatz, providing physical justification for it and indicating that the $n$-body operator $S_{n}$ is a true correlation operator for $n$ particle-hole (p-h) pairs. The ansatz is then used to decompose the $N$-body Schrödinger equation into a set of $N$ coupled, non-linear equations for the so-called subsystem amplitudes $S_{n}$, the $n$th equation for $S_{n}$ in the hierarchy being coupled to the other amplitudes $S_{i}$ $(1 \leqslant i \leqslant n+j)$ for a Hamiltonian containing up to $j$-body interaction terms. By iterating these equations one can make contact with perturbation theory by deriving quantities expressible in Goldstone diagrams.

In the fluid excited-state formalism one generally describes the true excited-state wavefunction as a linear combination of $1 \mathrm{p}-1 \mathrm{~h}, 2 \mathrm{p}-2 \mathrm{~h}, \ldots, N \mathrm{p}-\mathrm{Nh}$ excitations of the 
true ground state by introducing a new type of amplitude $S_{n}^{\mathrm{e}}$, similar to the ground state $S_{n}$. This ansatz is then used to decompose the excited-state $N$-body Schrödinger equation into a hierarchy of $N$ coupled, linear eigenvalue equations for the $S_{n}^{e}$ and excitation energies formally similar to the ground-state one.

Practical results require truncation of these hierarchies using, if possible, a physically motivated procedure. In this paper we employ the SUB $n$ truncation, where we set $S_{i}=0$ for $i>n$ in the ground-state formalism, and the $\operatorname{SUB}(m, n)$ truncation where we set $S_{i}^{\mathrm{e}}=S_{j}^{\mathrm{g}}=0$ for $i>m, j>n$, with superscripts e and $\mathrm{g}$ respectively referring to excited-and ground-state quantities in the excited-state formalism. We note that the SUB2 truncation is not simply Brueckner theory but much more, containing both it and most other well known approximations as well (Bishop and Lührmann 1978).

Besides having a very direct physical interpretation, the Ссм avoids the mixing of 'small', i.e. microscopic, terms with 'large' terms (Kümmel et al 1978), thereby preventing numerical instabilities and making the CCM computationally extremely efficient. Diagrammatically this is equivalent to removing the 'unlinked' terms from the Hamiltonian so that only 'linked' terms remain. These points and their physical background are discussed by Lahoz (1986).

The essence of the $\mathrm{CCM}$, in contrast for example with the configuration-interaction (CI) method (Nesbet 1958), lies with the increased degree of locality in the description of many-body correlation phenomena. The $\mathrm{CI}$ method contains unlinked diagrams for the energy and thereby suffers from the size-extensivity problem (Primas 1965, Bartlett and Purvis 1980, Kümmel 1984). This difficulty is fully resolved in the CCM. Together with the generalised time-ordering properties of the associated generalised tree diagrams, these features have proved to be extremely useful not only for large systems but also for systems with a small number of degrees of freedom (Kümmel et al 1978, Emrich 1981a, b, Kümmel 1984).

The CCM, extremely accurate by comparison with Green Function Monte Carlo (GFMC) calculations (Ceperley and Alder 1980, Bishop and Lührmann 1982, Emrich and Zabolitzky 1984), and providing more efficient numerical calculations than alternative methods (Bishop and Kümmel 1987), is ready for generalisation to two-component systems, where variational methods have been applied (Chakraborty and Pietiläinen 1982, Chakraborty et al 1983).

Section 2 of this paper concerns the methodology of the CCM emphasising the choice of model state and truncation scheme. Sections 3-5 are devoted to the ground state, where we treat the Schrödinger equation in a random-phase approximation (RPA) SUB2 calculation for potentials $V_{11}(q), V_{22}(q), V_{12}(q)$ such that their $q=0$ component, associated with long-range interactions, does not contribute. This involves using the theory of Muskhelishvili-Omnès integral equations to solve a set of coupled bilinear integral equations for the three-point functions associated with the problem. Crucial in this analysis is the role played by the dielectric function (cf Fetter and Walecka 1971 ) associated with each type of particle. For potentials satisfying $V_{11} V_{22}=V_{12}^{2}$, we obtain a unique solution and an expression for the correlation energy which we analyse in terms of the dimensionless mass ratio $M=m_{2} / m_{1}$. The cases $M=1, M=1836$, providing descriptions of an electron-hole system and a hydrogen plasma respectively, are investigated. The limit $M \rightarrow \infty$ is also taken (cf the Born-Oppenheimer (1927) approximation) to verify that our results make physical sense. For potentials satisfying $V_{11} V_{22} \neq V_{12}^{2}$, we obtain equations for the three-point functions and indicate how to obtain a formal solution. Sections 6 and 7 concern the excited states, where we derive the one- and two-body equations, treat the Schrödinger equation in an RPA-like 
$\operatorname{SUB}(1,2)$ calculation on the one-body equation, and obtain the relation, well known from two-body potential theory (Schiff 1968), linking the zeros of the ground-state RPA dielectric function to the poles of the relevant three-point function. Section 8 concerns conclusions and further work.

\section{Methodology}

Implementation of the $\mathrm{CCM}$ involves essentially two choices: (i) the inputs to the method, amounting largely to a choice of starting wavefunctions (model states); and (ii) the choice of decomposition of the equations into a natural hierarchy, amounting in practice to a particular approximation or truncation scheme. Although in principle these two choices are independent of each other, in practice they are intimately connected, for particular choices must depend not only on the individual physical system, but also on which of its possible phases is under study.

When solving the Schrödinger equation $H|\Psi\rangle=E|\Psi\rangle$, it is common practice to construct first a model state guided by physical and mathematical criteria and use it to obtain the true ground state by means of a perturbation-like procedure. This is the CCM approach where, having chosen a model state $|\Phi\rangle$, one makes the $\exp (S)$ ansatz, $|\Psi\rangle=\exp (S)|\Phi\rangle$, and attempts to solve $|\Psi\rangle$ in a particular approximation. Intuitively, the better $|\Phi\rangle$ is, the smaller the correlations $S_{n}$ need to be.

Clearly, the CCM equations must be truncated to obtain practical results. Three schemes have been well developed in the literature: (i) the SUB $n$ truncation (Bishop and Lührmann 1978, Emrich 1981a, b, Emrich and Zabolitzky 1984); (ii) the $\chi_{n}$ or Bochum truncation in closed-shell atomic nuclei (Zabolitzky 1974a, b, Kümmel et al 1978); (iii) the Offermann, Kümmel and Ey (OKE) approximation in open-shell atomic nuclei (Offermann et al 1976, Offermann 1975, 1976). Recently a super-SUB $n$ truncation has been developed to tackle ${ }^{4} \mathrm{He}$ (Robinson 1986).

In relatively low-density and/or weakly interacting systems, rarely do more than two or three particles lift themselves simultaneously out of the Fermi sea, which leads to the SUB $n$ truncation where all $S_{m}$ with $m>n$ are set to zero and the remaining $n$ coupled equations solved as accurately as possible. Similar considerations lead to the $\operatorname{SUB}(m, n)$ truncation in the excited states, where the ground- and excited-state equations are solved in the SUB $n$ and $\mathrm{SUB} m$ scheme respectively. In systems where the Coulombic interaction is weak (i.e. the correlation energy is small), $S_{3}$ and $S_{4}$ appear to be small correlations, which suggests that the method converges quickly (Kümmel 1984). On the other hand, recent work suggests that $S_{3}$ can have a nonnegligible effect (Szalewicz et al 1984, Haque and Kaldor 1987) if one wants extremely accurate results.

One can show that the fluid ground-state formalism corresponds to the overlap $\langle\Phi \mid \Psi\rangle \neq 0$ (cf Lahoz 1986), and that the fluid excited-state formalism corresponds to the overlap $\langle\Phi \mid \Psi\rangle=0$ (Emrich 1981a, b). Although we label these overlaps as the fluid ground and excited states, there is no certainty that the exact state obtained in the former $\left|\Psi_{\mathrm{g}}\right\rangle$ is the system's true ground state. All we are guaranteed is that it satisfies $\left\langle\Phi \mid \Psi_{\mathrm{g}}\right\rangle=1$; in particular our model state may be so ill-chosen that the state obtained bears no resemblance to the true ground state. This is illustrated by the work of Emrich (1981a, b), Stevens (1984) and Bishop (1985) who obtain states $\left|\Psi_{e}\right\rangle$ having energies lower than that described as the 'ground state'. The presence of these 'de-excited states' is entirely due to the choice of $|\Phi\rangle$ and suggests that a particular Fermi sea 
model state may not be the best choice. Amongst the ideas behind the choice of a new model state (Lahoz 1986) is that of symmetry breaking. In particular one can break particle number conservation as is done in BCs theory (Bardeen et al 1957). We break this symmetry when choosing $\left|\Phi_{\mathrm{BCS}}\right\rangle$ as our model state in paper II (Bishop and Lahoz 1987).

As the true ground state must either have zero or non-zero overlap with the model state, having both the above formalisms in principle enables one to find it. Further, by yielding 'de-excited' many-body states the excited-state formalism can provide a signal of any associated phase transition.

The richness of the CCM immediately becomes apparent even at the SUB2 level of approximation, where it is seen (Bishop and Lührmann 1978) to contain several well known approximations as subapproximations to itself: (i) the random-phase approximation RPA (Bohm and Pines 1953, Gell-Mann and Brueckner 1957) and its subapproximations the Tamm-Dancoff (TDA; Tamm 1945, Dancoff 1950) and Macke (1950) approximations; (ii) the Bethe-Goldstone (1957) approximation; (iii) the analogous Galitskii approximation (Mehta 1959, Chisholm and Squires 1959); and (iv) the standard Brueckner-Bethe-Goldstone theory (Fetter and Walecka 1971). The SUB2 is even richer than the fully self-consistent union of the above approximations as it contains extra classes of terms to preserve the overall antisymmetry required by Fermi-Dirac statistics. Thus it is not surprising that the full SUB2 approximation is algebraically complex. However, it is our belief that its underlying conceptual simplicity and the enormous amount of physics contained in it more than compensate for this complexity, and make the SUB2 (and CCM) a formidable technique. This SUB2 complexity makes it preferable to investigate those of its subapproximations susceptible to exact solution, in the hope that they will enable us to understand the wider problem and check subsequent numerical approximations. In this spirit we perform an RPA SUB2 treatment in the ground-state formalism and an RPA-like SUB $(1,2)$ treatment in the excited-state formalism.

A CCM problem can be tackled in several ways (Lahoz 1986). We investigate two-component Coulomb systems by choosing different model states to deal with Fermi fluids in paper I and Fermi superfluids in paper II.

\section{Derivation of RPA bilinear integral equations in the SUB2 approximation}

Consider a fermion system with two species of particles, ' 1 ' and ' 2 '. The system's Hamiltonian is

$$
H=\sum_{i=1,2}\left(\sum_{k=1}^{N_{i}} T_{i}\left(x_{k}\right)+\frac{1}{2} \sum_{k \neq l=1}^{N_{i}} V_{i i}\left(x_{k}, x_{l}\right)\right)+\sum_{k=1}^{N_{1}} \sum_{l=1}^{N_{2}} V_{12}\left(x_{k}, x_{l}\right)
$$

where $i$ refers to a particle of species $i, N_{i}$ to the number of particles of species $i$ with $N_{1}+N_{2}=N$, and $T$ and $V$ are kinetic and potential terms, respectively.

Consider the subsystem amplitudes $\Psi_{n}, n \leqslant N$ for a system of $N$ fermions with $n_{i}$ particles of species $i$ such that $n_{1}+n_{2}=n$, and with true ground-state wavefunction $|\Psi\rangle$. In terms of a model wavefunction $\left|\Phi_{F}\right\rangle$ built out of the $N$ orthonormalised single-particle states $\left|\nu_{1}\right\rangle, \ldots,\left|\nu_{N_{1}}\right\rangle ;\left|\mu_{1}\right\rangle, \ldots,\left|\mu_{N_{2}}\right\rangle \equiv\left|\lambda_{1}\right\rangle, \ldots,\left|\lambda_{N}\right\rangle$ we have

$$
\left|\Phi_{\mathrm{F}}\right\rangle=\prod_{i=1}^{N_{1}} \prod_{j=1}^{N_{2}} a_{\nu_{i}}^{+} a_{\mu_{j}}^{\dagger}|0\rangle
$$


where $|0\rangle$ is the vacuum state and the $a_{\nu_{i}}^{+}\left(a_{\mu_{1}}^{\dagger}\right)$ are a set of fermion creation operators for $\left|\nu_{i}\right\rangle\left(\left|\mu_{j}\right\rangle\right)$ which correspond to states normally occupied by species $1(2)$ with

$$
\left[a_{\nu_{1}}^{\dagger}, a_{\mu_{1}}^{+}\right]=0 \quad i=1, \ldots, N_{1} ; j=1, \ldots, N_{2} .
$$

We introduce the following terminology:

(i) $\alpha^{\prime}, \rho^{\prime}, \nu$ refer to particle-1 states;

(ii) $\beta^{\prime}, \sigma^{\prime}, \mu$ refer to particle-2 states;

(iii) $\gamma, \eta, \lambda$ refer to particle- 1 and/or 2 states;

(iv) $\lambda, \nu, \mu$ refer to states normally occupied (i.e. states filled in $\left.\left|\Phi_{F}\right\rangle\right)$;

(v) $\eta, \rho^{\prime}, \alpha^{\prime}$ refer to states normally unoccupied;

(vi) $\gamma, \alpha^{\prime}, \beta^{\prime}$ refer to both.

If there are only particles of type $i$ we have $\alpha^{\prime} \rightarrow \alpha, \rho^{\prime} \rightarrow \rho, \beta^{\prime} \rightarrow \beta, \sigma^{\prime} \rightarrow \sigma$.

The subsystem amplitudes are defined thus:

$$
\left\langle\gamma_{1} \ldots \gamma_{n}\left|\Psi_{n}\right| \lambda_{1} \ldots \lambda_{n}\right\rangle_{\mathrm{A}}=\left\langle\Phi_{\mathrm{F}}\left|a_{\lambda_{1}}^{\dagger} \ldots a_{\lambda_{n}}^{\dagger} a_{\gamma_{n}} \ldots a_{\gamma_{1}}\right| \Psi\right\rangle
$$

where $|\Psi\rangle$ is normalised by $\left\langle\Phi_{F} \mid \Psi\right\rangle=1$ and the subscript $A$ indicates an antisymmetrised state. This antisymmetrisation is to be performed only between states representing like particles.

Equation (3.4) can be interpreted as representing the amplitude that particles 'normally' in states $\lambda_{1}, \ldots, \lambda_{n}$ are in fact in states $\gamma_{1}, \ldots, \gamma_{n}$ with all other particles in their normally occupied states $\lambda_{n+1}, \ldots, \lambda_{N}$.

One can see (Lührmann 1977) that knowledge of $\Psi_{1}$ and $\Psi_{2}$ is sufficient to evaluate the ground-state energy $E=\left\langle\Phi_{\mathrm{F}}|H| \Psi\right\rangle=\left\langle\Phi_{\mathrm{F}}|(T+V)| \Psi\right\rangle$ :

$$
\begin{array}{r}
E=\sum_{\nu}\left\langle\nu\left|T_{1} \Psi_{1}\right| \nu\right\rangle+\sum_{\mu}\left\langle\mu\left|T_{2} \Psi_{1}\right| \mu\right\rangle+\frac{1}{2} \sum_{\nu \nu^{\prime}}\left\langle\nu \nu^{\prime}\left|V_{11} \Psi_{2}\right| \nu \nu^{\prime}\right\rangle_{\mathrm{A}} \\
+\frac{1}{2} \sum_{\mu \mu^{\prime}}\left\langle\mu \mu^{\prime}\left|V_{22} \Psi_{2}\right| \mu \mu^{\prime}\right\rangle_{\mathrm{A}}+\sum_{\nu \mu}\left\langle\nu \mu\left|V_{12} \Psi_{2}\right| \nu \mu\right\rangle
\end{array}
$$

where we assume that particles interact only via two-body potentials. The notation employed in (3.5) and hereafter implies that a complete set of states may be inserted whenever necessary, e.g.

$$
\left\langle\nu \nu^{\prime}\left|V_{11} \Psi_{2}\right| \nu \nu^{\prime}\right\rangle=\sum_{\alpha \alpha^{\prime}}\left\langle\nu \nu^{\prime}\left|V_{11}\right| \alpha \alpha^{\prime}\right\rangle\left\langle\alpha \alpha^{\prime}\left|\Psi_{2}\right| \nu \nu^{\prime}\right\rangle
$$

To derive the formal CCM equations consider the Schrödinger equation for the amplitudes $\Psi_{n}$ :

$$
\left\langle\Phi_{\mathrm{F}}\left|a_{\lambda_{1}}^{\dagger} \ldots a_{\lambda_{n}}^{\dagger} a_{\gamma_{n}} \ldots a_{\gamma_{1}} H\right| \Psi\right\rangle=E\left\langle\gamma_{1} \ldots \gamma_{n}\left|\Psi_{n}\right| \lambda_{1} \ldots \lambda_{n}\right\rangle_{\mathrm{A}} .
$$

We require a decomposition of the amplitudes $\Psi_{n}$ to eliminate all macroscopic terms (those proportional to $N$ ) in the essentially microscopic equations. To accomplish this we write

$$
|\Psi\rangle=\exp (S)\left|\Phi_{\mathrm{F}}\right\rangle
$$

where

$$
\begin{gathered}
S=\sum_{n=1}^{N} S_{n} \\
S_{n}=\left[\left(n_{1}\right) !\left(n_{2}\right) !\right]^{-2} \sum_{\substack{\eta_{1} \ldots \eta_{n} \\
\lambda_{1} \ldots \lambda_{n}}}\left\langle\eta_{1} \ldots \eta_{n}\left|S_{n}\right| \lambda_{1} \ldots \lambda_{n}\right\rangle_{A} a_{\eta_{1}}^{+} \ldots a_{\eta_{n}}^{+} a_{\lambda_{n}} \ldots a_{\lambda_{1}} .
\end{gathered}
$$


Insertion of equations (3.8) in (3.4) yields the decompositions:

$$
\begin{aligned}
& \left\langle\gamma\left|\Psi_{1}\right| \lambda\right\rangle=\langle\gamma \mid \lambda\rangle+\left\langle\gamma\left|S_{1}\right| \lambda\right\rangle \\
& \left\langle\gamma_{1} \gamma_{2}\left|\Psi_{2}\right| \lambda_{1} \lambda_{2}\right\rangle_{\mathrm{A}}=\left(\left\langle\gamma_{1}\left|\Psi_{1}\right| \lambda_{1}\right\rangle\left\langle\gamma_{2}\left|\Psi_{1}\right| \lambda_{2}\right\rangle\right)_{\mathrm{A}}+\left\langle\gamma_{1} \gamma_{2}\left|S_{2}\right| \lambda_{1} \lambda_{2}\right\rangle_{\mathrm{A}} \\
& \left\langle\gamma_{1} \gamma_{2} \gamma_{3}\left|\Psi_{3}\right| \lambda_{1} \lambda_{2} \lambda_{3}\right\rangle_{\mathrm{A}}=\left(\left\langle\gamma_{1}\left|\Psi_{1}\right| \lambda_{1}\right\rangle\left\langle\gamma_{2}\left|\Psi_{1}\right| \lambda_{2}\right\rangle\left\langle\gamma_{3}\left|\Psi_{1}\right| \lambda_{3}\right\rangle\right)_{\mathrm{A}} \\
& \quad+S_{123}\left(\left\langle\gamma_{1} \gamma_{2}\left|S_{2}\right| \lambda_{1} \lambda_{2}\right\rangle\left\langle\gamma_{3}\left|\Psi_{1}\right| \lambda_{3}\right\rangle\right)_{\mathrm{A}}+\left\langle\gamma_{1} \gamma_{2} \gamma_{3}\left|S_{3}\right| \lambda_{1} \lambda_{2} \lambda_{3}\right\rangle_{\mathrm{A}}
\end{aligned}
$$

where $S_{123}$ is the standard cyclic permutation operator and the antisymmetrisation is only to be performed on the ket states. Equation (3.9a) shows that $\left\langle\gamma\left|S_{1}\right| \lambda\right\rangle$ is that part of the amplitude $\left\langle\gamma\left|\Psi_{1}\right| \lambda\right\rangle$ not given by the single-particle amplitude $\langle\gamma \mid \lambda\rangle=\delta_{\gamma \lambda}$. As all other normally occupied states are in fact occupied, the Pauli principle dictates that the label $\gamma$ in $\left\langle\gamma\left|S_{1}\right| \lambda\right\rangle$ must equal some normally unoccupied label. Similar considerations apply to all correlation amplitudes $S_{n}$, which is reflected in the ansatz (3.8) where only normally unoccupied state labels appear in the bra labels. From equation $(3.9 b)\left\langle\left|S_{2}\right|\right\rangle$ is that part of the two-body subsystem amplitude $\left\langle\left|\Psi_{2}\right|\right\rangle$ which cannot be described in terms of one-body subsystem amplitudes $\left\langle\left|\Psi_{1}\right|\right\rangle$, which leads one to call it the two-body correlation amplitude.

To obtain the exact one- and two-body equations we must evaluate (3.7) (Lahoz 1986).

The one-body equation for a two-component fermion system is

$$
\begin{aligned}
\sum_{i=1}^{2}\left(\left\langle\gamma_{i}\left|T_{i} \Psi_{1}\right| \lambda_{i}\right\rangle\right. & +\sum_{j=1}^{2} \sum_{\lambda_{i}^{\prime}}\left\langle\gamma_{i} \lambda_{j}^{\prime}\left|T_{j}(2) S_{2}+V_{i j} \Psi_{2}\right| \lambda_{i} \lambda_{j}^{\prime}\right\rangle_{\mathrm{A}} \\
& +\frac{1}{2} \sum_{j=1}^{2} \sum_{k=j}^{2} \sum_{\lambda_{j}^{\prime} \lambda_{k}^{\prime \prime}}\left\langle\gamma_{i} \lambda_{j}^{\prime} \lambda_{k}^{\prime \prime}\left|\left(2-\delta_{j k}\right) V_{j k}(23) \chi_{3}^{(23)}\right| \lambda_{i} \lambda_{j}^{\prime} \lambda_{k}^{\prime \prime}\right\rangle_{\mathrm{A}} \\
& \left.-\sum_{\lambda_{i}^{\prime}} h_{\lambda_{i} \lambda_{i}}\left\langle\lambda_{i}\left|\Psi_{1}\right| \lambda_{i}^{\prime}\right\rangle\right)=0
\end{aligned}
$$

and the two-body equation is

$$
\begin{aligned}
\sum_{i=1}^{2} \sum_{j=i}^{2}\left\langle\left\langle\gamma_{i} \gamma_{j}^{\prime}\right|[\right. & \left.T_{i}(1)+T_{j}(2)\right] S_{2}+V_{i j} \Psi_{2}\left|\lambda_{i} \lambda_{j}^{\prime}\right\rangle_{\mathrm{A}} \\
& +\sum_{k=1}^{2} \sum_{\lambda_{k}^{\prime \prime}}\left\langle\gamma_{i} \gamma_{j}^{\prime} \lambda_{k}^{\prime \prime}\left|T_{k}(3) S_{3}+V_{i k}(13) \chi_{3}^{(13)}+V_{j k}(23) \chi_{3}^{(23)}\right| \lambda_{i} \lambda_{j}^{\prime} \lambda_{k}^{\prime \prime}\right\rangle_{\mathrm{A}} \\
& +\frac{1}{2} \sum_{\lambda_{i}^{\prime \prime} \lambda_{j}^{\prime \prime \prime}}\left\langle\gamma_{i} \gamma_{j}^{\prime}\left|S_{2}\right| \lambda_{i}^{\prime \prime} \lambda_{j}^{\prime \prime \prime}\right\rangle_{\mathrm{A}}\left\langle\lambda_{i}^{\prime \prime} \lambda_{j}^{\prime \prime \prime}\left|\left(2-\delta_{i j}\right) V_{i j}\right| \lambda_{i} \lambda_{j}^{\prime}\right\rangle_{\mathrm{A}} \\
& +\frac{1}{2} \sum_{k=1}^{2} \sum_{l=k}^{2} \sum_{\lambda_{k}^{\prime} \lambda^{\prime \prime \prime}}\left\langle\gamma_{i} \gamma_{j}^{\prime} \lambda_{k}^{\prime \prime} \lambda_{l}^{\prime \prime \prime}\left|\left(2-\delta_{k l}\right) V_{k l}(34) \chi_{4}^{(34)}\right| \lambda_{i} \lambda_{j}^{\prime} \lambda_{k}^{\prime \prime} \lambda_{l}^{\prime \prime \prime}\right\rangle_{\mathrm{A}} \\
& \left.-\sum_{k=1}^{2} \sum_{\lambda_{k}^{\prime \prime}}\left[\delta_{i k} h_{\lambda_{k}^{\prime} \lambda_{1}}\left\langle\gamma_{i} \gamma_{j}^{\prime}\left|S_{2}\right| \lambda_{k}^{\prime \prime} \lambda_{j}^{\prime}\right\rangle_{\mathrm{A}}+\delta_{j k} h_{\lambda_{k}^{\prime \prime} \lambda_{j}^{\prime}}\left\langle\gamma_{i} \gamma_{j}^{\prime}\left|S_{2}\right| \lambda_{i} \lambda_{k}^{\prime \prime}\right\rangle_{\mathrm{A}}\right]\right)=0
\end{aligned}
$$

where the index $i, \ldots, l$ represents particles of type $n$ when equal to $n$,

$$
\begin{gathered}
\chi_{3}^{(13)}=S_{2}(23) \Psi_{1}(1)+S_{2}(12) \Psi_{1}(3)+S_{3}(123) \\
\chi_{4}^{(34)}=S_{2}(13) S_{2}(24)+S_{2}(23) S_{2}(14)+S_{3}(123) \Psi_{1}(4)+S_{3}(124) \Psi_{1}(3)+S_{4}(1234) .
\end{gathered}
$$


Finally, the numbers in parentheses attached to the operators $T_{i}$ and $V_{i j}$ in equations (3.10) and (3.11) denote the quantum labels on the corresponding bra and ket counting from left to right on which the operator acts. The quantity $h_{\lambda, \lambda_{i}}$ in equations $(3.10)$ and (3.11) is termed the hole energy for particles of species $i$ and defined to be

$h_{\lambda_{r} \lambda_{i}^{\prime}}=\left\langle\lambda_{i}\left|T_{i} \Psi_{1}\right| \lambda_{i}^{\prime}\right\rangle+\sum_{\lambda_{i}^{\prime \prime}}\left\langle\lambda_{i} \lambda_{i}^{\prime \prime}\left(V_{i i} \Psi_{2}\left|\lambda_{i}^{\prime} \lambda_{i}^{\prime \prime}\right\rangle_{\mathrm{A}}+\sum_{\substack{\lambda_{j}^{\prime \prime} \\ j \neq i}}\left\langle\lambda_{i} \lambda_{j}^{\prime \prime}\left|V_{i j} \Psi_{2}\right| \lambda_{i}^{\prime} \lambda_{j}^{\prime \prime}\right\rangle\right.\right.$.

In equations (3.10) and (3.11) there are three types of $\left\langle\left|S_{2}\right|\right\rangle: S_{2}$ for species 1-1, 2-2, 1-2.

The general structure of the $n$-body equation is that the equation for $S_{n}$ involves $S_{n+1}$ and $S_{n+2}$. Clearly, in order to use the equations in practice a truncation scheme is necessary. We use the SUB2 approximation where all $S_{i}$ are set to zero for $i>2$.

Consider an infinite, homogeneous and overall neutral system of spin- $\frac{1}{2}$ particles consisting of two species, $i=1,2$, with masses $m_{i}$ and charges $e_{i}$. These particles interact via the following pairwise spin-independent local potentials: $V_{i i}$ between species $i-i, V_{12}$ between species 1-2, written in momentum space as follows:

$$
\begin{aligned}
& \left\langle\boldsymbol{k}_{i}, s_{i} ; \boldsymbol{k}_{i}^{\prime}, s_{i}^{\prime}\left|V_{i i}\right| \boldsymbol{k}_{i}^{\prime \prime}, s_{i}^{\prime \prime} ; \boldsymbol{k}_{i}^{\prime \prime \prime}, s_{i}^{\prime \prime \prime}\right\rangle=V_{i i}\left(\boldsymbol{k}_{i}-\boldsymbol{k}_{i}^{\prime \prime}\right) \delta_{\boldsymbol{k}_{i}+\boldsymbol{k}_{i,}^{\prime}, \boldsymbol{k}_{i}^{\prime \prime}+\boldsymbol{k}_{i}^{\prime \prime}} \delta_{s_{1} s_{i}^{\prime \prime}} \delta_{s_{i}^{\prime} s_{i}^{\prime \prime \prime}} \\
& \left\langle\boldsymbol{k}_{1}, s_{1} ; \boldsymbol{k}_{2}, s_{2}\left|V_{12}\right| \boldsymbol{k}_{1}^{\prime}, s_{1}^{\prime} ; \boldsymbol{k}_{2}^{\prime}, s_{2}^{\prime}\right\rangle=V_{12}\left(\boldsymbol{k}_{1}-\boldsymbol{k}_{1}^{\prime}\right) \delta_{\boldsymbol{k}_{1}+\boldsymbol{k}_{2}, \boldsymbol{k}_{1}^{\prime}+\boldsymbol{k}_{2}, \delta_{s_{1} s_{i}} \delta_{s_{2} s_{2}^{\prime}} .}
\end{aligned}
$$

Choose the single-particle basis to consist of plane-wave states with quantum labels

$$
\begin{array}{llll}
\nu \equiv\left(\boldsymbol{k}_{1}, s_{1}\right) & \left|\boldsymbol{k}_{1}\right|<k_{\mathrm{F}_{1}} & \mu \equiv\left(\boldsymbol{k}_{2}, s_{2}\right) & \left|\boldsymbol{k}_{2}\right|\left\langle k_{\mathrm{F}_{2}}\right. \\
\rho \equiv\left(\boldsymbol{k}_{1}, s_{1}\right) & \left|\boldsymbol{k}_{1}\right|>k_{\mathrm{F}_{1}} & \sigma \equiv\left(\boldsymbol{k}_{2}, s_{2}\right) & \left|\boldsymbol{k}_{2}\right|>k_{\mathrm{F}_{2}}
\end{array}
$$

where $s_{i}$ is a spin label with possible values $\uparrow$ or $\downarrow, k_{i}$ and $\hbar k_{\mathrm{F}_{1}}$ are the wavenumber and Fermi momentum respectively of the plane-wave states for species- $i$ particles. Introduce the convenient notation:

$$
\begin{aligned}
& X_{2 ; k_{1}}^{s_{1} s_{1}^{\prime}}(\boldsymbol{q}) \equiv\left\langle\boldsymbol{k}_{1}+q, s_{1} ; \boldsymbol{k}_{1}^{\prime}-\boldsymbol{q}, s_{1}^{\prime}\left|S_{2}\right| \boldsymbol{k}_{1}, s_{1} ; \boldsymbol{k}_{1}^{\prime}, s_{1}\right\rangle_{\mathrm{A}} \\
& Y_{2 ; \boldsymbol{k}_{2} k_{2}^{\prime}}^{s_{2} s_{2}^{\prime}}(\boldsymbol{q}) \equiv\left\langle\boldsymbol{k}_{2}+\boldsymbol{q}, s_{2} ; \boldsymbol{k}_{2}^{\prime}-\boldsymbol{q}, s_{2}^{\prime}\left|S_{2}\right| \boldsymbol{k}_{2}, s_{2} ; \boldsymbol{k}_{2}^{\prime}, s_{2}^{\prime}\right\rangle_{\mathrm{A}} \\
& Z_{2 ; \boldsymbol{k}_{1}}^{s_{1} s_{2}}(\boldsymbol{q}) \equiv\left\langle\boldsymbol{k}_{1}+\boldsymbol{q}, s_{1} ; \boldsymbol{k}_{2}-\boldsymbol{q}, s_{2}\left|S_{2}\right| \boldsymbol{k}_{1}, s_{1} ; \boldsymbol{k}_{2}, s_{2}\right\rangle
\end{aligned}
$$

where each of the three $\left\langle\left|S_{2}\right|\right\rangle$ can have the two (generally unequal) cases $S_{2}^{\uparrow \uparrow}=S_{2}^{\downarrow \downarrow}$ (parallel spins) and $S_{2}^{\uparrow \downarrow}=S_{2}^{\downarrow \uparrow}$ (antiparallel spins). By conservation of momentum $S_{1}=0$.

Implementing the SUB2 approximation in equation (3.11) gives the following expressions:

$$
\begin{aligned}
& \left(\hbar^{2} / 2 m_{1}\right)\left[\left|\boldsymbol{k}_{1}+\boldsymbol{q}\right|^{2}+\left|\boldsymbol{k}_{1}^{\prime}-\boldsymbol{q}\right|^{2}-\boldsymbol{k}_{1}^{2}-\boldsymbol{k}_{1}^{\prime 2}\right] X_{2 ; \boldsymbol{k}_{i} k_{i}}^{s_{1}^{\prime}}(\boldsymbol{q})+T_{\mathrm{RPA}}^{X}+T_{\mathrm{RPAEX}}^{X}+T_{\mathrm{CHP}}^{X} \\
& +T_{\mathrm{CPP}}^{X}+T_{\mathrm{CLAD}}^{X}+T_{\mathrm{PHA}}^{X}+T_{\mathrm{PHB}}^{X}+T_{\mathrm{EE} 1}^{X}+T_{\mathrm{EE} 2}^{X}=0 \\
& \left(\hbar^{2} / 2 m_{2}\right)\left[\left|\boldsymbol{k}_{2}+\boldsymbol{q}\right|^{2}+\left|\boldsymbol{k}_{2}^{\prime}-\boldsymbol{q}\right|^{2}-\boldsymbol{k}_{2}^{2}-\boldsymbol{k}_{2}^{\prime{ }^{2}}\right] Y_{2, \boldsymbol{k}_{2} \boldsymbol{k}_{2}^{\prime}}^{s_{2} s_{2}^{\prime}}(\boldsymbol{q})+T_{\mathrm{RPA}}^{Y}+\ldots+T_{\mathrm{EE} 2}^{Y}=0 \\
& \left\{\left(\hbar^{2} / 2 m_{1}\right)\left[\left|\boldsymbol{k}_{1}+\boldsymbol{q}\right|^{2}-\boldsymbol{k}_{1}^{2}\right]+\left(\hbar^{2} / 2 m_{2}\right)\left[\left|\boldsymbol{k}_{2}-\boldsymbol{q}\right|^{2}-\boldsymbol{k}_{2}^{2}\right]\right\} Z_{2 ; \boldsymbol{k}_{1} \boldsymbol{k}_{2}}^{s_{1} s_{2}}(\boldsymbol{q}) \\
& +T_{\mathrm{RPA}}^{Z}+T_{\mathrm{CHP}}^{Z}+\ldots+T_{\mathrm{EE} 2}^{Z}=0 \text {. }
\end{aligned}
$$


In equation (3.14) we have

$$
\begin{aligned}
& T_{\mathrm{RPA}}^{X}=V_{11}(q)\left(1+\sum_{\boldsymbol{k}_{1}^{\prime} \sigma_{1}} X_{2: k_{1}^{1} k_{1}^{\prime \prime}}^{s_{1} \sigma_{1}}(q) n\left(k_{1}^{\prime \prime}\right) \bar{n}\left(\boldsymbol{k}_{1}^{\prime \prime}-\boldsymbol{q}\right)\right) \\
& \times\left(1+\sum_{k_{1}^{\prime \prime} \sigma_{1}^{\prime}} X_{2 ; k_{1} k_{1}^{\prime \prime} k_{i}^{\prime}}^{\sigma_{1}^{\prime} s_{i}^{\prime}}(q) n\left(k_{1}^{\prime \prime \prime}\right) \bar{n}\left(\boldsymbol{k}_{1}^{\prime \prime \prime}+q\right)\right) \\
& +V_{12}(q)\left[\sum_{\boldsymbol{k}_{2}^{\prime \prime} \sigma_{2}} Z_{2 ; \boldsymbol{k}_{2}^{\prime \prime} \boldsymbol{k}_{i}}^{\sigma_{2} s^{\prime}}(\boldsymbol{q}) n\left(\boldsymbol{k}_{2}^{\prime \prime}\right) \bar{n}\left(\boldsymbol{k}_{2}^{\prime \prime}+\boldsymbol{q}\right)\right. \\
& \times\left(1+\sum_{\boldsymbol{k}_{1}^{\prime \prime} \sigma_{1}} X_{2 ; \boldsymbol{k}_{1} \boldsymbol{k}_{1}}^{s_{1} \sigma_{1}}(\boldsymbol{q}) n\left(\boldsymbol{k}_{1}^{\prime \prime}\right) \bar{n}\left(\boldsymbol{k}_{1}^{\prime \prime}-\boldsymbol{q}\right)\right) \\
& +\sum_{\boldsymbol{k}_{2}^{\prime \prime} \sigma_{2}} Z_{2 ; k_{1}^{2} k_{2}^{\prime \prime}}^{s_{1} \sigma_{2}}(\boldsymbol{q}) n\left(k_{2}^{\prime \prime}\right) \tilde{n}\left(\boldsymbol{k}_{2}^{\prime \prime}-\boldsymbol{q}\right) \\
& \left.\times\left(1+\sum_{\boldsymbol{k}_{1}^{\prime \prime} \sigma_{1}} X_{2 ; \boldsymbol{k}_{\mathrm{i}}^{\prime \prime} \boldsymbol{k}_{\mathrm{i}}}^{\sigma_{1} s_{i}^{\prime}}(\boldsymbol{q}) n\left(k_{1}^{\prime \prime}\right) \bar{n}\left(\boldsymbol{k}_{1}^{\prime \prime}+\boldsymbol{q}\right)\right)\right] \\
& +V_{22}(q) \sum_{\boldsymbol{k}_{2}^{\prime \prime} \sigma_{2}} \sum_{\boldsymbol{k}_{2}^{\prime \prime \prime} \sigma_{2}^{\prime}} Z_{2 ; k_{1}^{\prime} k_{2}^{\prime}}^{s_{1} \sigma_{2}}(q) Z_{2 ; k_{2}^{\prime \prime} k_{1}^{\prime}}^{\sigma_{2}^{\prime} s_{1}^{\prime}}(q) \\
& \times n\left(k_{2}^{\prime \prime}\right) n\left(k_{2}^{\prime \prime \prime}\right) \bar{n}\left(\boldsymbol{k}_{2}^{\prime \prime}-\boldsymbol{q}\right) \bar{n}\left(\boldsymbol{k}_{2}^{\prime \prime \prime}+\boldsymbol{q}\right)
\end{aligned}
$$

where $n\left(k_{i}\right) \equiv \theta\left(k_{\mathrm{F}}-k_{i}\right), \bar{n}\left(k_{i}\right) \equiv \theta\left(k_{i}-k_{\mathrm{F}}\right)$ and $\theta(x)$ is the step function, defined to equal one (zero) for $x$ greater (less) than zero. The remaining terms, where we employ the one-component case nomenclature, can be found in Lahoz (1986).

Using the convention of conservation of total momentum and third component of spin at every vertex and that arrows pointing upwards (downwards) denote 'particle states' ('hole states') associated with normally unoccupied (occupied) states with momenta outside (inside) the Fermi sphere, plus the definitions

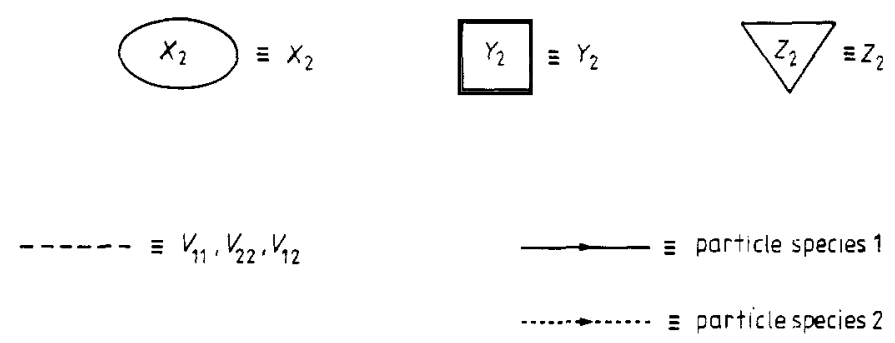

we can associate diagrams with equation (3.17). These are given by figure 1. Figures 2 and 3 are associated with the remaining terms for which explicit equations may be similarly derived.

We now solve (3.14)-(3.16) in the RPA:

$$
\begin{aligned}
& \left(\hbar^{2} / 2 m_{i}\right)\left[\left|\boldsymbol{k}_{i}+\boldsymbol{q}\right|^{2}+\left|\boldsymbol{k}_{i}^{\prime}-\boldsymbol{q}\right|^{2}-\boldsymbol{k}_{i}^{2}-\boldsymbol{k}_{i}^{\prime 2}\right] S_{2 ; \boldsymbol{k}_{1} \boldsymbol{k}_{i}^{\prime}}^{s_{1}^{\prime}}(\boldsymbol{q})=-T_{\mathrm{RPA}}^{S} \\
& \quad i=1, S=X ; i=2, S=Y \\
& \left\{\left(\hbar^{2} / 2 m_{1}\right)\left[\left|\boldsymbol{k}_{1}+\boldsymbol{q}\right|^{2}-\boldsymbol{k}_{1}^{2}\right]+\left(\hbar^{2} / 2 m_{2}\right)\left[\left|\boldsymbol{k}_{2}-\boldsymbol{q}\right|^{2}-\boldsymbol{k}_{2}^{2}\right]\right\} Z_{2 ; \boldsymbol{k}_{1}^{\prime} \boldsymbol{k}_{2}}^{s_{1} s_{2}}(\boldsymbol{q})=-T_{\mathrm{RPA}}^{Z}
\end{aligned}
$$




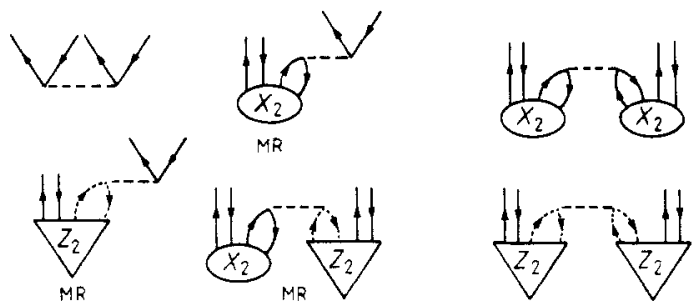

Figure 1. The diagrammatic representation of the RPA terms of equation (3.17). The terms which have MR underneath them represent two separate terms, one as shown and the other obtained by mirror reflection.
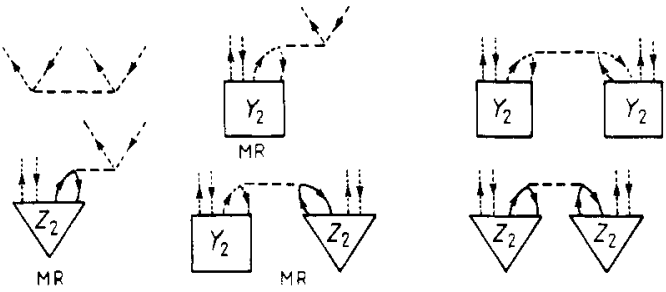

Figure 2. Same as figure 1 but for $T_{\mathrm{RPA}}^{Y}$.

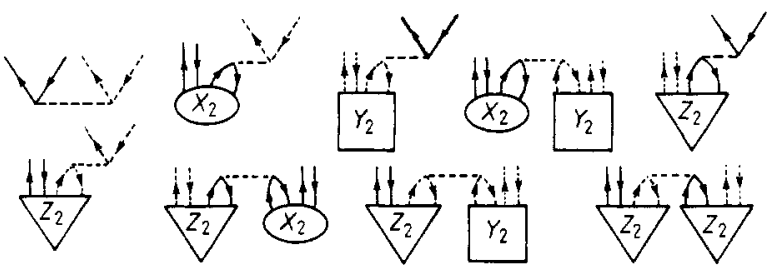

Figure 3. Same as figure 1 but for $T_{\mathrm{RPA}}^{Z}$.

Diagrammatically, the equation for $S=X$ has the representation given by figure 4 . Similar representations apply to equation (3.18) with $S=Y, Z$. Clearly, the above RPA equations are responsible for generating the two-component analogue of the usual one-component ring diagrams.

We introduce the functions

$$
\begin{aligned}
& f_{k_{1}}^{11}(q) \equiv \sum_{-k_{i}^{i} \in \Gamma_{1}} X_{2 ; k_{1} k_{i}}(q) \\
& f_{\boldsymbol{k}_{2}}^{22}(\boldsymbol{q}) \equiv \sum_{-\boldsymbol{k}_{2}^{\prime} \in \Gamma_{2}} Y_{2 ; \boldsymbol{k}_{2} \boldsymbol{k}_{2}}(\boldsymbol{q}) \\
& f_{k_{1}}^{12}(\boldsymbol{q}) \equiv \sum_{-\boldsymbol{k}_{2} \in \Gamma_{2}} Z_{2 ; \boldsymbol{k}_{1} \boldsymbol{k}_{2}}(\boldsymbol{q})
\end{aligned}
$$

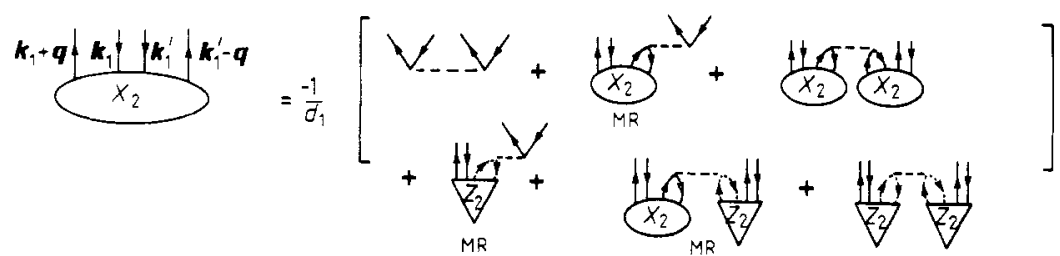

Figure 4. The diagrammatic representation of equation (3.18) with $S=X$ and $d_{1}=$ $\left(\hbar^{2} / m_{1}\right)\left[\boldsymbol{q} \cdot\left(\boldsymbol{k}_{1}-\boldsymbol{k}_{1}^{\prime}+\boldsymbol{q}\right)\right]$. 
where the $\left\langle\left|S_{2}\right|\right\rangle$ have been assumed spin independent, i.e. $S_{2}^{\uparrow}=S_{2}^{\uparrow \downarrow}=S_{2}$ (as is valid in the RPA but not in the exact solution), the first (second) superscript denotes the open (closed) legs of the relevant four-point function $\left\langle\left|S_{2}\right|\right\rangle$, and the integration regions $\Gamma_{i}$ are defined by

$$
\boldsymbol{k}_{i} \in \Gamma_{i} \Rightarrow\left\{\begin{array}{l}
\left|\boldsymbol{k}_{i}\right|<k_{\mathrm{F}_{1}} \\
\left|\boldsymbol{k}_{i}+\boldsymbol{q}\right|>k_{\mathrm{F}_{i}}
\end{array}\right.
$$

Use of the equality $X_{2 ; \boldsymbol{k}_{i}}(q) \equiv X_{2 ;-\boldsymbol{k}_{i},-\boldsymbol{k}_{1}}(q)$ which follows from the defining relation and Galilean invariance and its counterparts for $Y_{2}, Z_{2}$ yields

$$
\begin{aligned}
& f_{-k_{1}^{\prime}}^{11}(q) \equiv \sum_{\boldsymbol{k}_{1} \in \Gamma_{1}} X_{2 ; \boldsymbol{k}_{1} \boldsymbol{k}_{1}}(q) \\
& f_{-\boldsymbol{k}_{2}^{\prime}}^{22}(\boldsymbol{q}) \equiv \sum_{\boldsymbol{k}_{2} \in \Gamma_{2}} Y_{2 ; \boldsymbol{k}_{2} \boldsymbol{k}_{2}}(q) \\
& f_{-\boldsymbol{k}_{2}}^{21}(\boldsymbol{q}) \equiv \sum_{\boldsymbol{k}_{1} \in \Gamma_{1}} Z_{2 ; \boldsymbol{k}_{1} \boldsymbol{k}_{2}}(\boldsymbol{q}) .
\end{aligned}
$$

Substitution of equations (3.19) and (3.21) in equation (3.18) for $S=X$ yields

$$
\begin{aligned}
& \left(\hbar^{2} / 2 m_{1}\right) \boldsymbol{q} \cdot\left(\boldsymbol{k}_{1}-\boldsymbol{k}_{1}^{\prime}+\boldsymbol{q}\right) X_{2 ; \boldsymbol{k}_{1} \boldsymbol{k}_{1}^{\prime}}(\boldsymbol{q})=-V_{11}(q)\left[1+2 f_{k_{1}}^{11}(\boldsymbol{q})\right]\left[1+2 f_{-k_{1}^{\prime}}^{11}(\boldsymbol{q})\right] \\
& -2 V_{12}(q)\left[f_{k_{1}}^{12}(q)+f_{-k_{i}}^{12}(q)\right]-4 V_{12}(q)\left[f_{-k_{i}}^{12}(q) f_{k_{1}}^{11}(q)+f_{k_{1}}^{12}(q) f_{-k_{i}}^{11}(q)\right] \\
& -4 V_{22}(q) f_{k_{1}}^{12}(q) f_{-k_{1}^{\prime}}^{12}(q) \text {. }
\end{aligned}
$$

We find it convenient to introduce the three-point functions:

$$
\begin{array}{ll}
g_{q}\left(\boldsymbol{k}_{1}\right) \equiv g\left(\boldsymbol{k}_{1}\right) \equiv 1+2 f_{k_{1}}^{11}(\boldsymbol{q}) & \hat{g}_{\boldsymbol{q}}\left(\boldsymbol{k}_{1}\right) \equiv \hat{\boldsymbol{g}}\left(\boldsymbol{k}_{1}\right) \equiv 2 f_{k_{1}}^{12}(\boldsymbol{q}) \\
h_{\boldsymbol{q}}\left(\boldsymbol{k}_{2}\right) \equiv h\left(\boldsymbol{k}_{2}\right) \equiv 1+2 f_{\boldsymbol{k}_{2}}^{22}(\boldsymbol{q}) & \hat{h}_{\boldsymbol{q}}\left(\boldsymbol{k}_{2}\right) \equiv \hat{h}\left(\boldsymbol{k}_{2}\right) \equiv 2 f_{\boldsymbol{k}_{2}}^{21}(\boldsymbol{q}) .
\end{array}
$$

Substituting (3.23) in (3.22) gives

$$
\begin{aligned}
X_{2 ; \boldsymbol{k}_{1} \boldsymbol{k}_{1}^{\prime}}(\boldsymbol{q})=- & \frac{m_{1}}{\hbar^{2}} \frac{1}{\boldsymbol{q} \cdot\left(\boldsymbol{k}_{1}-\boldsymbol{k}_{1}^{\prime}+\boldsymbol{q}\right)} \\
& \times\left\{\boldsymbol{g}\left(\boldsymbol{k}_{1}\right)\left[V_{11} g\left(-\boldsymbol{k}_{1}^{\prime}\right)+V_{12} \hat{g}\left(-\boldsymbol{k}_{1}^{\prime}\right)\right]+\hat{g}\left(\boldsymbol{k}_{1}\right)\left[V_{12} g\left(-\boldsymbol{k}_{1}^{\prime}\right)+V_{22} \hat{g}\left(-\boldsymbol{k}_{1}^{\prime}\right)\right]\right\}
\end{aligned}
$$

where for ease of notation we drop the $q$ dependence of the potentials. Summing both sides of (3.24) over $-\boldsymbol{k}_{1}^{\prime} \in \Gamma_{1}$, using equations (3.21) and (3.23), changing the sum to an integral in the standard manner and introducing the more symmetric momentum variables $\boldsymbol{\kappa}_{i}, \boldsymbol{\kappa}_{i}^{\prime}$ by

$$
\boldsymbol{k}_{i}=\boldsymbol{\kappa}_{i}-\frac{1}{2} \boldsymbol{q} \quad \boldsymbol{k}_{i}^{\prime}=-\boldsymbol{\kappa}_{i}^{\prime}+\frac{1}{2} \boldsymbol{q}
$$

yields the integral equation

$$
g\left(\boldsymbol{\kappa}_{1}\right)=1-\frac{1}{2 \pi \beta_{0}}\left(g\left(\boldsymbol{\kappa}_{1}\right) \int_{\Gamma_{i}^{\prime}} \mathrm{d} \boldsymbol{\kappa}_{1}^{\prime} \frac{G_{1}\left(\boldsymbol{\kappa}_{1}^{\prime}\right)}{\boldsymbol{q} \cdot\left(\boldsymbol{\kappa}_{1}^{\prime}+\boldsymbol{\kappa}_{1}\right)}+\hat{g}\left(\boldsymbol{\kappa}_{1}\right) \int_{\Gamma_{i}^{\prime}} \mathrm{d} \boldsymbol{\kappa}_{1}^{\prime} \frac{G_{2}\left(\boldsymbol{\kappa}_{1}^{\prime}\right)}{\boldsymbol{q} \cdot\left(\boldsymbol{\kappa}_{1}^{\prime}+\boldsymbol{\kappa}_{1}\right)}\right)
$$

where we have defined

$$
\begin{aligned}
& \beta_{0} \equiv 2 \pi^{2} \hbar^{2} / m_{1} \Omega \\
& \boldsymbol{\kappa}_{i} \in \Gamma_{i}^{\prime} \Rightarrow\left\{\begin{array}{l}
\left|\boldsymbol{\kappa}_{i}-\frac{1}{2} \boldsymbol{q}\right|<k_{\mathrm{F}_{i}} \\
\left|\boldsymbol{\kappa}_{i}+\frac{1}{2} \boldsymbol{q}\right|>k_{\mathrm{F}_{i}}
\end{array}\right. \\
& g\left(\boldsymbol{k}_{1}\right)=g\left(\boldsymbol{\kappa}_{1}-\frac{1}{2} \boldsymbol{q}\right) \equiv g\left(\boldsymbol{\kappa}_{1}\right) \quad \hat{g}\left(\boldsymbol{k}_{1}\right)=\hat{g}\left(\boldsymbol{\kappa}_{1}-\frac{1}{2} \boldsymbol{q}\right) \equiv \hat{g}\left(\boldsymbol{\kappa}_{1}\right) \\
& X_{2 ; \boldsymbol{\kappa}_{i} \boldsymbol{k}_{i}}(\boldsymbol{q})=X_{2 ; \boldsymbol{\kappa}_{1}-\boldsymbol{q} / 2,-\boldsymbol{\kappa}_{i}+\boldsymbol{q} / 2}(\boldsymbol{q}) \equiv X_{2 ; \boldsymbol{\kappa}_{1} \boldsymbol{\kappa}_{\mathrm{i}}}(\boldsymbol{q}) \\
& G_{1}\left(\boldsymbol{\kappa}_{1}\right) \equiv V_{11} g\left(\boldsymbol{\kappa}_{1}\right)+V_{12} \hat{g}\left(\boldsymbol{\kappa}_{1}\right) \quad G_{2}\left(\boldsymbol{\kappa}_{1}\right) \equiv V_{12} g\left(\boldsymbol{\kappa}_{1}\right)+V_{22} \hat{g}\left(\boldsymbol{\kappa}_{1}\right) .
\end{aligned}
$$


Similar analyses on equation (3.18) for $S=Y$ and $Z$ yield

$h\left(\boldsymbol{\kappa}_{2}\right)=1-\frac{M}{2 \pi \beta_{0}}\left(h\left(\boldsymbol{\kappa}_{2}\right) \int_{\Gamma_{2}^{\prime}} \mathrm{d} \boldsymbol{\kappa}_{2}^{\prime} \frac{H_{1}\left(\boldsymbol{\kappa}_{2}^{\prime}\right)}{\boldsymbol{q} \cdot\left(\boldsymbol{\kappa}_{2}^{\prime}+\boldsymbol{\kappa}_{2}\right)}+\hat{h}\left(\boldsymbol{\kappa}_{2}\right) \int_{\Gamma_{2}^{\prime}} \mathrm{d} \boldsymbol{\kappa}_{2}^{\prime} \frac{H_{2}\left(\boldsymbol{\kappa}_{2}^{\prime}\right)}{\boldsymbol{q} \cdot\left(\boldsymbol{\kappa}_{2}^{\prime}+\boldsymbol{\kappa}_{2}\right)}\right)$

$\hat{g}\left(\boldsymbol{\kappa}_{1}\right)=\frac{-M}{2 \pi \beta_{0}}\left(g\left(\boldsymbol{\kappa}_{1}\right) \int_{\Gamma_{2}^{\prime}} \mathrm{d} \boldsymbol{\kappa}_{2}^{\prime} \frac{H_{2}\left(\boldsymbol{\kappa}_{2}^{\prime}\right)}{q \cdot\left(\boldsymbol{\kappa}_{2}^{\prime}+M \boldsymbol{\kappa}_{1}\right)}+\hat{g}\left(\boldsymbol{\kappa}_{1}\right) \int_{\Gamma_{2}^{\prime}} \mathrm{d} \boldsymbol{\kappa}_{2}^{\prime} \frac{H_{1}\left(\boldsymbol{\kappa}_{2}^{\prime}\right)}{q \cdot\left(\boldsymbol{\kappa}_{2}^{\prime}+M \boldsymbol{\kappa}_{1}\right)}\right)$

$\hat{h}\left(\boldsymbol{\kappa}_{2}\right)=\frac{-1}{2 \pi \beta_{0}}\left(h\left(\boldsymbol{\kappa}_{2}\right) \int_{\Gamma_{i}^{\prime}} \mathrm{d} \boldsymbol{\kappa}_{1}^{\prime} \frac{G_{2}\left(\boldsymbol{\kappa}_{1}^{\prime}\right)}{q \cdot\left(\boldsymbol{\kappa}_{1}^{\prime}+\boldsymbol{\kappa}_{2} / M\right)}+\hat{h}\left(\boldsymbol{\kappa}_{2}\right) \int_{\Gamma_{i}^{\prime}} \mathrm{d} \boldsymbol{\kappa}_{1}^{\prime} \frac{G_{1}\left(\boldsymbol{\kappa}_{1}^{\prime}\right)}{q \cdot\left(\boldsymbol{\kappa}_{1}^{\prime}+\boldsymbol{\kappa}_{2} / M\right)}\right)$

where we have defined

$$
\begin{aligned}
& M \equiv m_{2} / m_{1} \\
& H_{1}\left(\boldsymbol{\kappa}_{2}\right) \equiv V_{22} h\left(\boldsymbol{\kappa}_{2}\right)+V_{12} \hat{h}\left(\boldsymbol{\kappa}_{2}\right) \\
& H_{2}\left(\boldsymbol{\kappa}_{2}\right) \equiv V_{12} h\left(\boldsymbol{\kappa}_{2}\right)+V_{11} \hat{h}\left(\boldsymbol{\kappa}_{2}\right) .
\end{aligned}
$$

Equations (3.26)-(3.29), which are four non-linear coupled integral equations for the four RPA particle-hole vertex functions $g\left(\kappa_{1}\right), \ldots, h\left(\kappa_{2}\right)$, are the basic two-component CCM RPA equations since all other quantities of interest may be obtained from them. As we solve the RPA in terms of the three-point functions $G_{1}\left(\kappa_{1}\right), \ldots, H_{2}\left(\kappa_{2}\right)$ two cases arise:

(i) $V_{11} V_{22}=V_{12}^{2}$-the Coulombic potential case

(ii) $V_{11} V_{22} \neq V_{12}^{2}$-the non-Coulombic potential case

which are related to the matrix of the potentials:

$$
V=\left[\begin{array}{ll}
V_{11} & V_{12} \\
V_{12} & V_{22}
\end{array}\right]
$$

being singular or non-singular.

The mathematical techniques involved in solving both cases are essentially the same; however, in the former the equations simplify. The non-Coulombic case analysis is inapplicable to Coulombic potentials as the matrix $\mathbf{V}$ becomes singular.

\section{RPA treatment of the Coulombic potential case}

In the Coulombic potential case, i.e. $V_{11} V_{22}=V_{12}^{2}$, we have

$$
G_{2}\left(\kappa_{1}\right)=\left(V_{12} / V_{11}\right) G_{1}\left(\kappa_{1}\right) \quad H_{2}\left(\kappa_{2}\right)=\left(V_{12} / V_{22}\right) H_{1}\left(\kappa_{2}\right)
$$

and it is sufficient to solve for $G_{1}\left(\boldsymbol{\kappa}_{1}\right)$ and $H_{1}\left(\boldsymbol{\kappa}_{2}\right)$.

Combining (3.26)-(3.29) with (4.1) yields

$$
\begin{aligned}
& G_{1}\left(\boldsymbol{\kappa}_{1}\right)=V_{11}-\frac{G_{1}\left(\boldsymbol{\kappa}_{1}\right)}{2 \pi \beta}\left(\int_{\tilde{\Gamma}_{1}} \mathrm{~d} \boldsymbol{\kappa}_{1}^{\prime} \frac{G_{1}\left(\boldsymbol{\kappa}_{1}^{\prime}\right)}{\boldsymbol{q} \cdot\left(\boldsymbol{\kappa}_{1}^{\prime}+\boldsymbol{\kappa}_{1}\right)}+\int_{\tilde{\Gamma}_{2}} \mathrm{~d} \boldsymbol{\kappa}_{2}^{\prime} \frac{M H_{1}\left(\boldsymbol{\kappa}_{2}^{\prime}\right)}{\boldsymbol{q} \cdot\left(\boldsymbol{\kappa}_{2}^{\prime}+\boldsymbol{M} \boldsymbol{\kappa}_{1}\right)}\right) \\
& H_{1}\left(\boldsymbol{\kappa}_{2}\right)=V_{22}-\frac{H_{1}\left(\boldsymbol{\kappa}_{2}\right)}{2 \pi \beta}\left(\int_{\tilde{\Gamma}_{2}} \mathrm{~d} \boldsymbol{\kappa}_{2}^{\prime} \frac{M H_{1}\left(\boldsymbol{\kappa}_{2}^{\prime}\right)}{\boldsymbol{q} \cdot\left(\boldsymbol{\kappa}_{2}^{\prime}+\boldsymbol{\kappa}_{2}\right)}+\int_{\tilde{\Gamma}_{1}} \mathrm{~d} \boldsymbol{\kappa}_{1}^{\prime} \frac{G_{1}\left(\boldsymbol{\kappa}_{1}^{\prime}\right)}{\boldsymbol{q} \cdot\left(\boldsymbol{\kappa}_{1}^{\prime}+\boldsymbol{\kappa}_{2} / M\right)}\right)
\end{aligned}
$$


where we have rendered all momenta dimensionless by writing them in terms of $k_{F_{1}}$; $V_{i i}=V_{i i}\left(q k_{\mathrm{F}_{1}}\right)$ and have defined

$$
\begin{aligned}
& \beta \equiv \beta_{0} / k_{\mathrm{F}_{1}} \\
& \boldsymbol{\kappa}_{1} \in \tilde{\Gamma}_{1} \Rightarrow\left\{\begin{array} { l } 
{ | \boldsymbol { \kappa } _ { 1 } - \frac { 1 } { 2 } \boldsymbol { q } | < 1 } \\
{ | \boldsymbol { \kappa } _ { 1 } + \frac { 1 } { 2 } \boldsymbol { q } | > 1 }
\end{array} \quad \boldsymbol { \kappa } _ { 2 } \in \tilde { \Gamma } _ { 2 } \Rightarrow \left\{\begin{array}{l}
\left|\boldsymbol{\kappa}_{2}-\frac{1}{2} \boldsymbol{q}\right|<k_{\mathrm{F}_{2}} / k_{\mathrm{F}_{1}} \\
\left|\boldsymbol{\kappa}_{2}+\frac{1}{2} \boldsymbol{q}\right|>k_{\mathrm{F}_{2}} / k_{\mathrm{F}_{1}} .
\end{array}\right.\right.
\end{aligned}
$$

Equations (4.2) and (4.3) are exactly linearised by employing a generalisation of the trick used in the one-component case (Bishop and Lührmann 1978). First we multiply (4.2) by $\left[\boldsymbol{q} \cdot\left(\boldsymbol{\kappa}_{1}+\boldsymbol{\kappa}_{1}^{\prime \prime}\right)\right]^{-1}$ and integrate with respect to $\boldsymbol{\kappa}_{1} \in \tilde{\Gamma}_{1}$; then we multiply (4.3) by $\left[\boldsymbol{q} \cdot\left(\boldsymbol{\kappa}_{2}+M \boldsymbol{\kappa}_{1}^{\prime \prime}\right)\right]^{-1}$ and integrate with respect to $\boldsymbol{\kappa}_{2} \in \tilde{\Gamma}_{2}$.

The above integrations and use of equations (4.2) and (4.3) yields the functional relation

$$
\frac{V_{11}^{2}}{G_{1}\left(\kappa_{1}\right) G_{1}\left(-\kappa_{1}\right)}=K\left(\kappa_{1}\right)
$$

where the known function $K\left(\boldsymbol{\kappa}_{1}\right)$ is defined by the relation

$$
\begin{aligned}
K\left(\boldsymbol{\kappa}_{1}\right) \equiv 1+\frac{1}{2 \pi \beta}\left[\int_{\tilde{\Gamma}_{1}} \mathrm{~d} \boldsymbol{\kappa}_{1}^{\prime}\left(\frac{1}{q \cdot\left(\boldsymbol{\kappa}_{1}^{\prime}+\boldsymbol{\kappa}_{1}\right)}+\frac{1}{q \cdot\left(\boldsymbol{\kappa}_{1}^{\prime}-\boldsymbol{\kappa}_{1}\right)}\right)\right. \\
\left.\quad+\int_{\tilde{\Gamma}_{2}} \mathrm{~d} \boldsymbol{\kappa}_{2}^{\prime} M\left(\frac{1}{q \cdot\left(\boldsymbol{\kappa}_{2}^{\prime}+M \boldsymbol{\kappa}_{1}\right)}+\frac{1}{q \cdot\left(\boldsymbol{\kappa}_{2}^{\prime}-M \boldsymbol{\kappa}_{1}\right)}\right)\right] .
\end{aligned}
$$

A similar analysis yields the functional relation

$$
\frac{V_{22}^{2}}{H_{1}\left(\boldsymbol{\kappa}_{2}\right) H_{1}\left(-\boldsymbol{\kappa}_{2}\right)}=L\left(\boldsymbol{\kappa}_{2}\right)
$$

where $L\left(\kappa_{2}\right)$ is defined by $L\left(\kappa_{2}\right) \equiv K\left(\kappa_{2} / M\right)$. Substitution of (4.4) in (4.2) and (4.6) in (4.3) yields the linear equations

$$
\begin{aligned}
G_{1}\left(\boldsymbol{\kappa}_{1}\right) K\left(\boldsymbol{\kappa}_{1}\right)= & V_{11}\left(1+\frac{1}{2 \pi \beta} \int_{\tilde{\Gamma}_{1}} \mathrm{~d} \boldsymbol{\kappa}_{1}^{\prime} \frac{G_{1}\left(\boldsymbol{\kappa}_{1}^{\prime}\right)}{q \cdot\left(\boldsymbol{\kappa}_{1}^{\prime}-\boldsymbol{\kappa}_{1}\right)}\right. \\
& \left.+\frac{M}{2 \pi \beta} \int_{\tilde{\Gamma}_{2}} \mathrm{~d} \boldsymbol{\kappa}_{2}^{\prime} \frac{H_{1}\left(\boldsymbol{\kappa}_{2}^{\prime}\right)}{\boldsymbol{q} \cdot\left(\boldsymbol{\kappa}_{2}^{\prime}-M \boldsymbol{\kappa}_{1}\right)}\right) \\
H_{1}\left(\boldsymbol{\kappa}_{2}\right) L\left(\boldsymbol{\kappa}_{2}\right)= & V_{22}\left(1+\frac{M}{2 \pi \beta} \int_{\tilde{\Gamma}_{2}} \mathrm{~d} \boldsymbol{\kappa}_{2}^{\prime} \frac{H_{1}\left(\boldsymbol{\kappa}_{2}^{\prime}\right)}{\boldsymbol{q} \cdot\left(\boldsymbol{\kappa}_{2}^{\prime}-\boldsymbol{\kappa}_{2}\right)}\right. \\
& \left.+\frac{1}{2 \pi \beta} \int_{\tilde{\Gamma}_{1}} \mathrm{~d} \boldsymbol{\kappa}_{1}^{\prime} \frac{G_{1}\left(\boldsymbol{\kappa}_{1}^{\prime}\right)}{q \cdot\left(\boldsymbol{\kappa}_{1}^{\prime}-\boldsymbol{\kappa}_{2} / M\right)}\right)
\end{aligned}
$$

Decoupling of the above equations is now accomplished by introducing the integral equations

$$
\begin{aligned}
& I_{0}\left(\boldsymbol{\kappa}_{1}\right)=1+\frac{V_{11} I_{0}\left(\boldsymbol{\kappa}_{1}\right)}{2 \pi \beta} \int_{\tilde{\Gamma}_{1}} \mathrm{~d} \boldsymbol{\kappa}_{1}^{\prime} \frac{1}{I_{0}\left(\boldsymbol{\kappa}_{1}^{\prime}\right) K\left(\boldsymbol{\kappa}_{1}^{\prime}\right) \boldsymbol{q} \cdot\left(\boldsymbol{\kappa}_{1}^{\prime}-\boldsymbol{\kappa}_{1}\right)} \\
& J_{0}\left(\boldsymbol{\kappa}_{2}\right)=1+\frac{V_{22} J_{0}\left(\boldsymbol{\kappa}_{2}\right)}{2 \pi \beta} \int_{\tilde{\Gamma}_{2}} \mathrm{~d} \boldsymbol{\kappa}_{2}^{\prime} \frac{M}{J_{0}\left(\boldsymbol{\kappa}_{2}^{\prime}\right) L\left(\boldsymbol{\kappa}_{2}^{\prime}\right) \boldsymbol{q} \cdot\left(\boldsymbol{\kappa}_{2} \boldsymbol{\kappa}_{2}^{\prime}-\boldsymbol{\kappa}_{2}\right)}
\end{aligned}
$$


(which are linear in $I_{0}^{-1}$ and $J_{0}^{-1}$ respectively) and using the one-component case trick (Bishop and Lührmann 1978). We multiply (4.7) by $\left[K\left(\boldsymbol{\kappa}_{1}\right) I_{0}\left(\boldsymbol{\kappa}_{1}\right) \boldsymbol{q} \cdot\left(\boldsymbol{\kappa}_{1}-\boldsymbol{\kappa}_{2}^{\prime \prime} / M\right)\right]^{-1}$ and integrate with respect to $\kappa_{1} \in \tilde{\Gamma}_{1}$, and multiply $(4.8)$ by $\left[L\left(\kappa_{2}\right) J_{0}\left(\kappa_{2}\right) q \cdot\left(\kappa_{2}-\right.\right.$ $\left.\left.M \kappa_{1}^{\prime \prime}\right)\right]^{-1}$ and integrate with respect to $\kappa_{2} \in \tilde{\Gamma}_{2}$. The first integration yields after using (4.8) and (4.9):

$\hat{G}_{1}\left(\boldsymbol{\kappa}_{1}\right) K\left(\boldsymbol{\kappa}_{1}\right)=V_{11}+\frac{V_{11}}{2 \pi \beta} \int_{\tilde{\Gamma}_{1}} \mathrm{~d} \boldsymbol{\kappa}_{1}^{\prime} \frac{\hat{G}_{1}\left(\boldsymbol{\kappa}_{1}^{\prime}\right)}{\boldsymbol{q} \cdot\left(\boldsymbol{\kappa}_{1}^{\prime}-\boldsymbol{\kappa}_{1}\right)} \quad \boldsymbol{\kappa}_{1} \in \tilde{\Gamma}_{1} \quad M \boldsymbol{\kappa}_{1} \in \tilde{\Gamma}_{2}$.

Similarly, the second integration yields

$$
\hat{H}_{1}\left(\boldsymbol{\kappa}_{2}\right) L\left(\boldsymbol{\kappa}_{2}\right)=V_{22}+\frac{V_{22}}{2 \pi \beta} \int_{\tilde{\Gamma}_{2}} \mathrm{~d} \boldsymbol{\kappa}_{2}^{\prime} \frac{M \hat{H}_{1}\left(\boldsymbol{\kappa}_{2}^{\prime}\right)}{\boldsymbol{q} \cdot\left(\boldsymbol{\kappa}_{2}^{\prime}-\boldsymbol{\kappa}_{2}\right)} \quad \boldsymbol{\kappa}_{2} \in \tilde{\Gamma}_{2} \quad \boldsymbol{\kappa}_{2} / M \in \tilde{\Gamma}_{1}
$$

where we have introduced the functions $\hat{G}_{1}\left(\boldsymbol{\kappa}_{1}\right), \hat{H}_{1}\left(\boldsymbol{\kappa}_{2}\right)$ defined to be

$$
\begin{aligned}
& \hat{G}_{1}\left(\boldsymbol{\kappa}_{1}\right) \equiv G_{1}\left(\boldsymbol{\kappa}_{1}\right) / J_{0}\left(\boldsymbol{M} \boldsymbol{\kappa}_{1}\right) \\
& \hat{H}_{1}\left(\boldsymbol{\kappa}_{2}\right) \equiv H_{1}\left(\boldsymbol{\kappa}_{2}\right) / I_{0}\left(\boldsymbol{\kappa}_{2} / M\right) .
\end{aligned}
$$

It can be seen from equations (4.11) and (4.12) that the two-component RPA Coulombic potential case reduces to linear integral equations possessing the same structure as their one-component counterpart (Bishop and Lührmann 1978), the solution of which is well known.

Consider the case where all three potentials are bare Coulombic:

$$
V_{i i}(q)=\frac{4 \pi e_{i}^{2}}{\Omega q^{2}} \quad V_{12}(q)=\frac{4 \pi e_{1} e_{2}}{\Omega q^{2}} \quad q \neq 0
$$

Omission of the $q=0$ case is physically justified as this contribution from the potentials can be shown to cancel by means of an appropriate convergence factor.

Introduce the following dimensionless parameters:

$$
r_{\mathrm{s}}=r_{1} / a_{1} \quad \alpha \equiv(4 / 9 \pi)^{1 / 3}
$$

where $k_{\mathrm{F}_{i}}=\left(3 \pi^{2} \rho_{i}\right)^{1 / 3}, \rho_{i}$ is the particle number density for species $i, r_{i}$ is the average interparticle spacing of species $i$ defined by $\rho_{i}=\left(4 \pi r_{i}^{3} / 3\right)^{-1}$ and $a_{i}$ is the Bohr radius of species $i$ defined by $a_{i}=\hbar^{2} / m_{i} e_{i}^{2}$, and make the simplifying assumptions $k_{\mathrm{F}_{1}}=k_{\mathrm{F}_{2}}=$ $k_{\mathrm{F}} ; e_{1}=-e_{2}=e$, so that

$$
\boldsymbol{\kappa}_{i} \in \tilde{\Gamma}_{i} \Rightarrow\left\{\begin{array}{l}
\left|\boldsymbol{\kappa}_{i}-\frac{1}{2} \boldsymbol{q}\right|<1 \\
\left|\boldsymbol{\kappa}_{i}+\frac{1}{2} \boldsymbol{q}\right|>1
\end{array}\right.
$$

Equations (4.11) and (4.12) are linear but singular in the integration regimes $\kappa_{i} \in \tilde{\Gamma}_{i}$, so their kernels are not square integrable and the usual Fredholm theory of linear integral equations is inapplicable.

However, solution of (4.11) and (4.12) is possible provided the correct coordinate system is chosen. Analysis of (4.11) and (4.12) indicates that $\hat{G}_{1}\left(\kappa_{1}\right), \hat{H}_{1}\left(\kappa_{2}\right)$ are 
to us are $\kappa^{2}, q^{2}$ and $\boldsymbol{\kappa} \cdot q$. As $K\left(\kappa_{1}\right)$ is independent of $\kappa_{1}^{2}$, and we regard $q$ as a parameter, equations (4.11) and (4.12) have a solution $\hat{G}_{1}\left(\boldsymbol{\kappa}_{1}\right)=\hat{G}_{1}\left(\boldsymbol{\kappa}_{1} \cdot \hat{\boldsymbol{q}}\right), \hat{H}_{1}\left(\boldsymbol{\kappa}_{2}\right)=$ $\hat{H}_{1}\left(\kappa_{2} \cdot \hat{q}\right)$. Clearly, the natural coordinate system in which to solve (4.11) and (4.12) is a cylindrical polar system with axis along the direction $\hat{q}$ and, for obvious reasons of symmetry, with the origin at the midpoint of the axis joining the centres of the unit Fermi spheres implied by the integration regime $\tilde{\Gamma}_{i}$.

In these cylindrical polar coordinates $\left(x_{i}, \rho_{i}, \theta_{i}\right)$ where $x_{i} \equiv \boldsymbol{\kappa}_{i} \cdot \hat{q}$, the integrations expressed in equations (4.5), (4.6), (4.11) and (4.12) can be written (Lahoz 1986)

$$
\begin{aligned}
& \int_{\tilde{\Gamma}_{i}} \mathrm{~d} \boldsymbol{\kappa}_{i} f\left(x_{i}\right)=2 \pi q \int_{\tilde{L}_{i}} \mathrm{~d} x_{i} \tilde{N}_{i}\left(x_{i}\right) f\left(x_{i}\right) \\
& \tilde{N}_{i}\left(x_{i}>0\right)= \begin{cases}x_{i} & 0 \leqslant x_{i} \leqslant 1-\frac{1}{2} q, q<2 \\
{\left[1-\left(\frac{1}{2} q-x_{i}\right)^{2}\right] / 2 q} & 1-\frac{1}{2} q \leqslant x_{i} \leqslant 1+\frac{1}{2} q, q<2 \\
{\left[1-\left(\frac{1}{2} q-x_{i}\right)^{2}\right] / 2 q} & \frac{1}{2} q-1 \leqslant x_{i} \leqslant \frac{1}{2} q+1, q \geqslant 2 \\
0 & \text { otherwise }\end{cases} \\
& x_{i} \in M \tilde{L}_{i} \Rightarrow \begin{cases}0 \leqslant x_{i} \leqslant M\left(\frac{1}{2} q+1\right) & q<2 \\
M\left(\frac{1}{2} q-1\right) \leqslant x_{i} \leqslant M\left(\frac{1}{2} q+1\right) & q \geqslant 2\end{cases}
\end{aligned}
$$

for an arbitrary function $f\left(x_{i}\right)$.

Writing (4.11) and (4.12) in cylindrical polar coordinates strongly suggests the need to make an appeal to analytic continuation to solve the resulting equations. This we do, and defining functions of a complex variable $K\left(z_{1}\right), L\left(z_{2}\right)$ :

$$
\begin{aligned}
K\left(z_{1}\right)=1+\frac{1}{\tilde{\beta}} & {\left[\int_{\tilde{L}_{1}} \mathrm{~d} x_{1}^{\prime} \tilde{N}_{1}\left(x_{1}^{\prime}\right)\left(\frac{1}{x_{1}^{\prime}+z_{1}}+\frac{1}{x_{1}^{\prime}-z_{1}}\right)\right.} \\
& \left.+\int_{L_{2}} \mathrm{~d} x_{2}^{\prime} M \tilde{N}_{2}\left(x_{2}^{\prime}\right)\left(\frac{1}{x_{2}^{\prime}+M z_{1}}+\frac{1}{x_{2}^{\prime}-M z_{1}}\right)\right] \\
& L\left(z_{2}\right)=K\left(z_{2} / M\right) \quad \tilde{\beta} \equiv \beta / V_{11}\left(q k_{\mathrm{F}}\right)
\end{aligned}
$$

we can define functions of a complex variable $\hat{G}_{1}\left(z_{1}\right), \hat{H}_{1}\left(z_{2}\right)$ which satisfy

$$
\begin{aligned}
K\left(z_{1}\right) \hat{G}_{1}\left(z_{1}\right)= & 1+\frac{1}{\tilde{\beta}} \int_{\tilde{L}_{1}} \mathrm{~d} x_{1}^{\prime} \frac{\left[\tilde{N}_{1}\left(x_{1}^{\prime}\right)+M \tilde{N}_{2}\left(M x_{1}^{\prime}\right)\right] \hat{G}_{1}\left(x_{1}^{\prime}\right)}{x_{1}^{\prime}-z_{1}} \\
& \hat{G}_{1}\left(x_{1}\right)=\lim _{y_{1} \rightarrow 0} \hat{G}_{1}\left(x_{1} \pm \mathrm{i} y_{1}\right) \quad x_{1} \in \tilde{L}_{1}, M x_{1} \in \tilde{L}_{1} \\
L\left(z_{2}\right) \hat{H}_{1}\left(z_{2}\right)= & 1+\frac{M}{\tilde{\beta}} \int_{M \tilde{L}_{2}} \mathrm{~d} x_{2}^{\prime} \frac{\left[\tilde{N}_{2}\left(x_{2}^{\prime}\right)+\tilde{N}_{1}\left(x_{2}^{\prime} / M\right) / M\right] \hat{H}_{1}\left(x_{2}^{\prime}\right)}{x_{2}^{\prime}-z_{2}} \\
& \hat{H}_{1}\left(x_{2}\right)=\lim _{y_{2} \rightarrow 0} \hat{H}_{1}\left(x_{2} \pm \mathrm{i} y_{2}\right) \quad x_{2} \in \tilde{L}_{2}, x_{2} / M \in \tilde{L}_{2} .
\end{aligned}
$$

Converting (4.17) and (4.18) into Muskhelishvili-Omnès equations (1953, 1958) and using the one-component results (Bishop and Lührmann 1978) yields the general solutions (Lahoz 1986):

$$
\begin{aligned}
& G_{1}\left(z_{1}\right)=V_{11} \exp \left[u\left(z_{1}\right)\right]\left[1+P\left(z_{1}\right)\right] J_{0}\left(M z_{1}\right) / K\left(z_{1}\right) \\
& H_{1}\left(z_{2}\right)=V_{22} \exp \left[v\left(z_{2}\right)\right]\left[1+Q\left(z_{2}\right)\right] I_{0}\left(z_{2} / M\right) / L\left(z_{2}\right)
\end{aligned}
$$


where

$$
\begin{gathered}
u\left(z_{1}\right) \equiv \frac{1}{2 \pi \mathrm{i}} \int_{L_{1}} \mathrm{~d} x_{1}^{\prime} \frac{1}{x_{1}^{\prime}-z_{1}} \operatorname{Ln}\left(1-\frac{2 \pi \mathrm{i}\left[\tilde{N}_{1}\left(x_{1}^{\prime}\right)+M \tilde{N}_{2}\left(M x_{1}^{\prime}\right)\right]}{\tilde{\beta} K_{+}\left(x_{1}^{\prime}\right)}\right)^{-1} \\
v\left(z_{2}\right) \equiv \frac{1}{2 \pi \mathrm{i}} \int_{M \tilde{L}_{2}} \mathrm{~d} x_{2}^{\prime} \frac{1}{x_{2}^{\prime}-z_{2}} \operatorname{Ln}\left(1-\frac{2 \pi \mathrm{i} M\left[\tilde{N}_{2}\left(x_{2}^{\prime}\right)+\tilde{N}_{1}\left(x_{2}^{\prime} / M\right) / M\right]}{\tilde{\beta} L_{+}\left(x_{2}^{\prime}\right)}\right)^{-1} \\
K_{ \pm}\left(x_{1}\right)=\lim _{\varepsilon \rightarrow 0} K\left(x_{1} \pm \mathrm{i} \varepsilon\right) \\
L_{ \pm}\left(x_{2}\right)=K_{ \pm}\left(x_{2} / M\right)
\end{gathered}
$$

and $P\left(z_{1}\right)\left(Q\left(z_{2}\right)\right)$ are arbitrary functions analytic in the entire complex $z_{1}\left(z_{2}\right)$ plane with the possible exceptions of singularities at either or both of the ends of $\tilde{L}_{1}\left(M \tilde{L}_{2}\right)$. $\mathrm{Ln}$ indicates any value of the multivalued logarithmic function which is continuous on $\tilde{L}_{1}\left(M \tilde{L}_{2}\right)$.

By making use of the functional relations (4.4)-(4.6) in general form:

$$
\begin{aligned}
& \frac{V_{11}^{2}}{G_{1}\left(z_{1}\right) G_{1}\left(-z_{1}\right)}=K\left(z_{1}\right) \\
& \frac{V_{22}^{2}}{H_{1}\left(z_{2}\right) H_{1}\left(-z_{2}\right)}=L\left(z_{2}\right)
\end{aligned}
$$

we now show that for all finite $M \geqslant 1$ a unique solution for $G_{1}\left(z_{1}\right)$ and $H_{1}\left(z_{2}\right)$ exists. Having obtained solutions for finite $M$ we consider the case where $M$ becomes infinitely large (the Born-Oppenheimer approximation).

To obtain these unique solutions we require knowledge of the analytic structure of the functions $\operatorname{Ln} K\left(z_{1}\right)$ and $\operatorname{Ln} L\left(z_{2}\right)$ (Lahoz 1986), for which purpose it remains only to analyse the zeros of $K\left(z_{1}\right)$ and $L\left(z_{2}\right)$.

Clearly $K\left(z_{1}\right)$ is analytic in the entire complex $z_{1}$ plane except for cuts along $z_{1} \in\left(\tilde{L}_{1}+\tilde{L}_{1}^{\prime}\right)$ where $x_{1} \in \tilde{L}_{1} \Leftrightarrow-x_{1} \in \tilde{L}_{1}^{\prime}$. The only possible zeros of $K\left(z_{1}\right)$ are seen to be on the real axis outside the cuts $\tilde{L}_{1}, \tilde{L}_{1}^{\prime}$ and are at $z_{1}= \pm x_{1}^{\mathrm{R}}$ where $x_{1}^{\mathrm{R}}$ is the positive root (if any) of the equation

$$
-1=\frac{\alpha r_{\mathrm{s}}}{\pi q^{3}}\left[K^{(1)}\left(x_{1}^{\mathrm{R}}\right)+M K^{(1)}\left(M x_{1}^{\mathrm{R}}\right)\right] \quad x_{1}^{\mathrm{R}}>\frac{1}{2} q+1
$$

where

$$
\begin{aligned}
K^{(1)}\left(x_{1}\right) \equiv 2 q & +\left[1-\left(\frac{1}{2} q+x_{1}\right)^{2}\right] \ln \left|\frac{x_{1}+\frac{1}{2} q+1}{x_{1}+\frac{1}{2} q-1}\right| \\
& +\left[1-\left(\frac{1}{2} q-x_{1}\right)^{2}\right] \ln \left|\frac{x_{1}-\frac{1}{2} q-1}{x_{1}-\frac{1}{2} q+1}\right| .
\end{aligned}
$$

One can show that (4.24) has either one solution or no solution, according as $q \leqslant q_{1}$ or $q>q_{1}$ respectively, where $q_{1}$ is given by

$$
x_{1}^{\mathrm{R}}\left(q_{1}\right)=\frac{1}{2} q_{1}+1 \text {. }
$$

Consider the behaviour of $K_{+}\left(x_{1}\right)$ as $M$ is increased continuously from $M=1$. It can 
be shown (Lahoz 1986), and figures 5-11 render this clear, that as $M$ increases the dominant contribution to $\operatorname{Re} K_{+}\left(x_{1}\right)$ comes from $\left(\alpha r_{s} / \pi q^{3}\right) M K^{(1)}\left(M x_{1}\right)$ in the region $0<x_{1}<x_{1}^{(M)}$ and from $\left(\alpha r_{\mathrm{s}} / \pi q^{3}\right) K^{(1)}\left(x_{1}\right)$ in the region $x_{1}>x_{1}^{(M)}$ where $x_{1}^{(M)} \sim \mathrm{O}\left(M^{-\delta}\right)$, $\delta>0$. Similar behaviour is observed for $\operatorname{Im} K_{+}\left(x_{1}\right)$.

More importantly we see that for $q<q_{1}$ the real phase $\delta_{1}\left(x_{1}\right)$ defined by

$$
K_{ \pm}\left(x_{1}\right)=\left|K_{ \pm}\left(x_{1}\right)\right| \exp \left( \pm \mathrm{i} \delta_{1}\left(x_{1}\right)\right)
$$

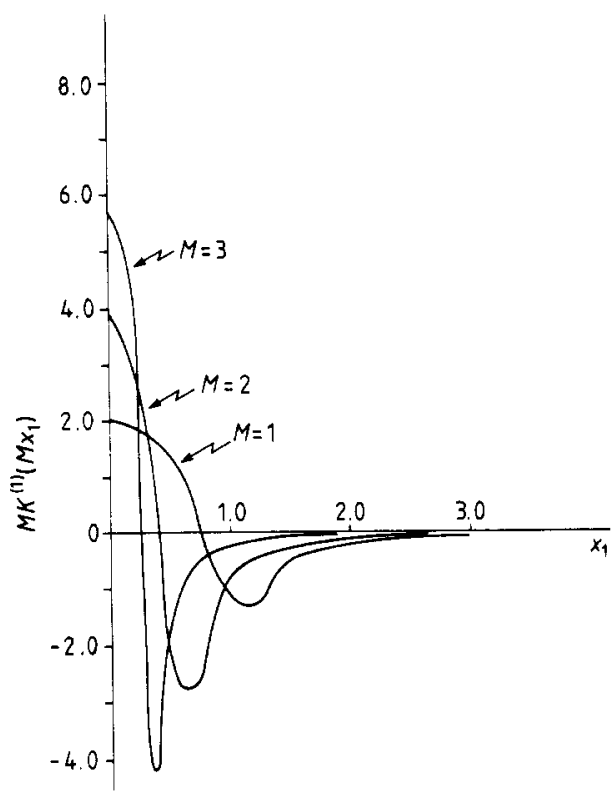

Figure 5. Plot of $M K^{(1)}\left(M x_{1}\right)$ against $x_{1}$ for values of the mass ratio $M$ equal to $1,2,3\left(q=0.5, r_{\mathrm{s}}=1\right)$.

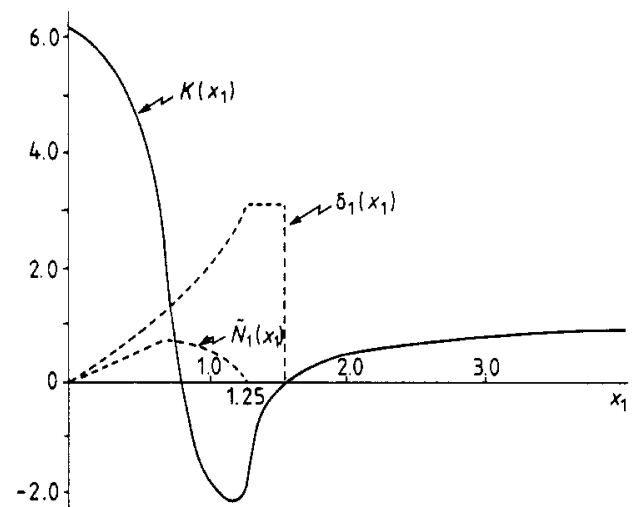

Figure 6. Plot of $\operatorname{Re} K_{+}\left(x_{1}\right)=K\left(x_{1}\right), \operatorname{Im} K_{+}\left(x_{1}\right)=$ $\left(4 \alpha r_{s} / q^{2}\right) \tilde{N}_{1}\left(x_{1}\right)$, and the phase angle $\delta_{1}\left(x_{1}\right)$ for mass ratio $M=1, r_{\mathrm{s}}=1$ and momentum transfer $q=0.5<$ $q_{\max }=0.729$, where $q_{1}=q_{2}=q_{\max }$.

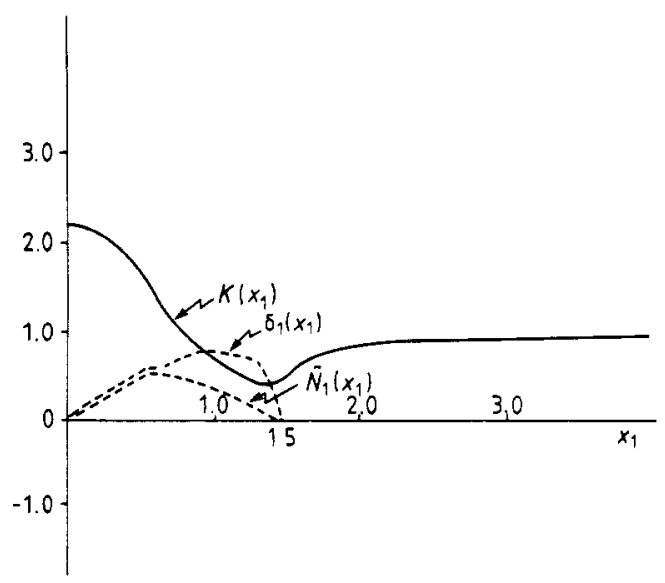

Figure 7. Same as figure 6 but at a momentum transfer $q=1.0>q_{\max }=0.729$. 


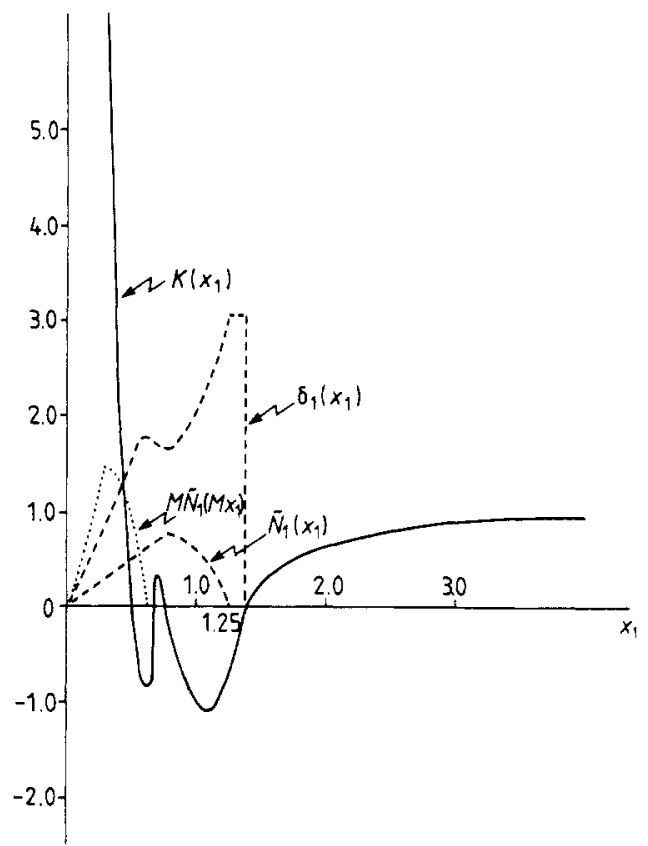

Figure 8. Plot of $\operatorname{Re} K_{+}\left(x_{1}\right)=\boldsymbol{K}\left(x_{1}\right), \operatorname{Im} K_{+}=$ $\left(2 \alpha r_{\mathrm{s}} / q^{2}\right)\left[\tilde{N}(x)+M \tilde{N}_{1}\left(M x_{1}\right)\right]$ and the phase angle $\delta_{1}\left(x_{1}\right)$ for mass ratio $M=2, r_{\mathrm{s}}=1$ and momentum transfer $q=0.5<q_{1}$, where $x_{1}^{\mathrm{R}}\left(q_{1}\right)=\frac{1}{2} q_{1}+1$.

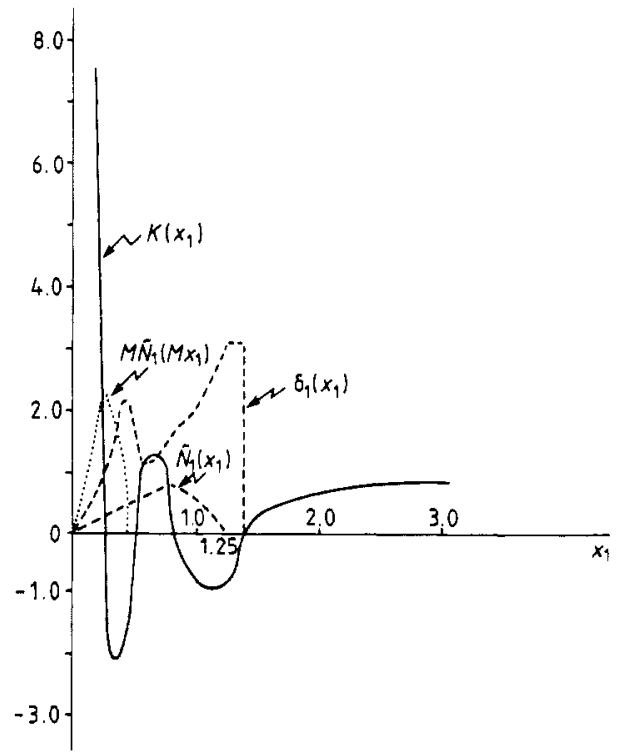

Figure 10. Same as figure 8 but with mass ratio $M=3$.

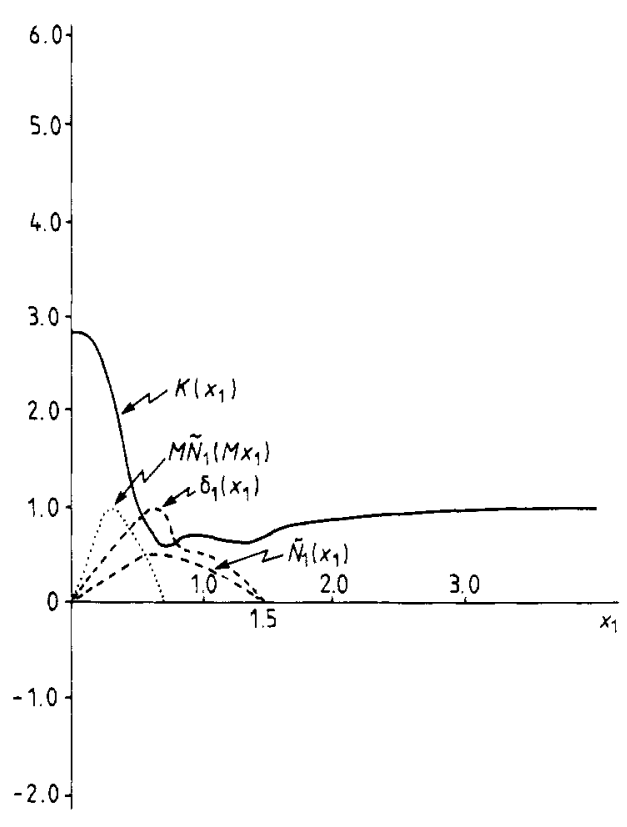

Figure 9. Same as figure 8 but at a momentum transfer $q=1.0>q_{1}$.

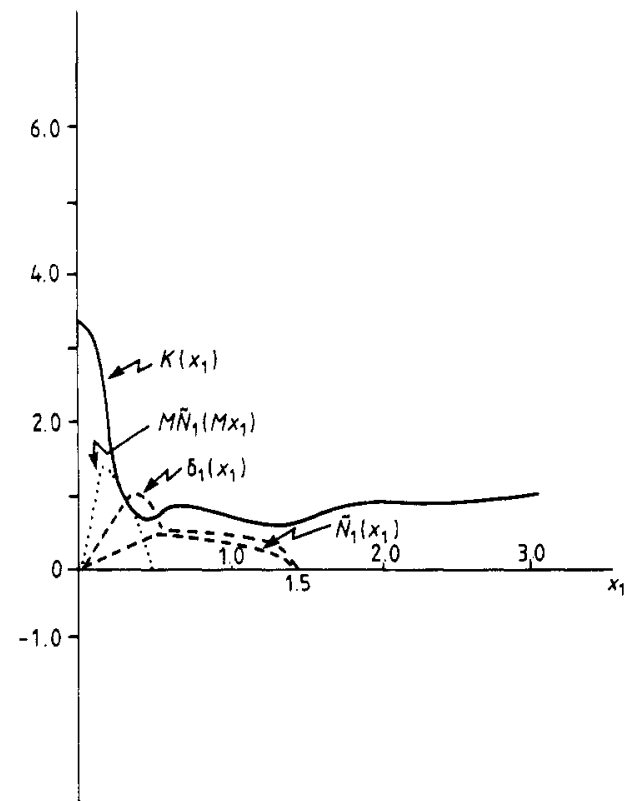

Figure 11. Same as figure 8 but with mass ratio $M=3$ and at momentum transfer $q=1.0>q_{1}$. 
or, equivalently,

$$
\delta_{1}\left(x_{1}\right)=-\delta_{1}\left(-x_{1}\right)=\operatorname{Tan}^{-1}\left(\operatorname{Im} K_{+}\left(x_{1}\right) / \operatorname{Re} K_{+}\left(x_{1}\right)\right)
$$

where $\operatorname{Tan}^{-1}$ indicates in general any value of the multivalued inverse tangent function which is continuous on $\tilde{L}_{1}$, is equal (for the particular choice where $\delta_{1}\left(x_{1}\right) \rightarrow 0$ as $x_{1} \rightarrow \infty$ ) to $\pi$ in the region $\frac{1}{2} q+1 \leqslant x_{1}<x_{1}^{\mathrm{R}}$, whereas for $q>q_{1}$ it is equal to 0 for $x_{1} \geqslant \frac{1}{2} q+1$. This behaviour is connected with the respective presence or absence of a zero at $x_{1}= \pm x_{1}^{\mathrm{R}}$ in the function $K\left(x_{1}\right)$.

The behaviour of the function $L\left(z_{2}\right)$ is rendered clear by figures $12-16$. We see again that for $q<q_{2}$ the real phase $\delta_{2}\left(x_{2}\right)$ defined by

$$
L_{ \pm}\left(x_{2}\right)=\left|L_{ \pm}\left(x_{2}\right)\right| \exp \left( \pm \mathrm{i} \delta_{2}\left(x_{2}\right)\right)
$$

where $x_{2}^{\mathrm{R}}\left(q_{2}\right)=M\left(\frac{1}{2} q_{2}+1\right)$ and satisfies (4.24) with $x_{1}^{\mathrm{R}}=x_{2}^{\mathrm{R}} / M$, or, equivalently,

$$
\delta_{2}\left(x_{2}\right)=-\delta_{2}\left(-x_{2}\right)=\operatorname{Tan}^{-1}\left(\operatorname{Im} L_{+}\left(x_{2}\right) / \operatorname{Re} L_{+}\left(x_{2}\right)\right)
$$

is equal to $\pi$ in the region $M\left(\frac{1}{2} q+1\right) \leqslant x_{2} \leqslant x_{2}^{\mathrm{R}}$, whereas for $q>q_{2}$ it is equal to 0 for $x_{2} \geqslant M\left(\frac{1}{2} q+1\right)$.

It is straightforward to show that (Lahoz 1986)

$$
\begin{aligned}
G_{1}\left(z_{1}\right) G_{1}\left(-z_{1}\right) & =V_{11}^{2}\left[1+P\left(z_{1}\right)\right]\left[1+P\left(-z_{1}\right)\right] J_{0}\left(M z_{1}\right) J_{0}\left(-M z_{1}\right) \\
& \times \frac{1}{K^{2}\left(z_{1}\right)} \exp \left(\frac{1}{2 \pi \mathrm{i}} \int_{L_{1}+\tilde{L}_{\mathrm{i}}^{\prime}} \mathrm{d} x_{1}^{\prime} \frac{\ln \left[K_{+}\left(x_{1}^{\prime}\right) / K_{-}\left(x_{1}^{\prime}\right)\right]}{x_{1}^{\prime}-z_{1}}\right) .
\end{aligned}
$$

The integral in (4.31) can be simplified by considering a suitable contour integral

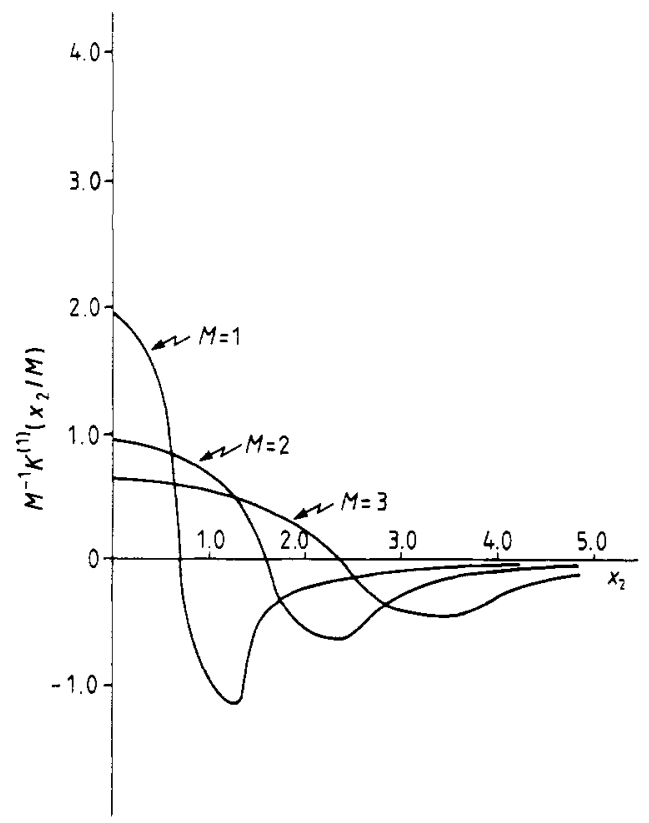

Figure 12. Plot of $K^{(1)}\left(x_{2} / M\right) / M$ against $x_{2}$ for values of the mass ratio $M$ equal to 1,2 and $3\left(q=0.5, r_{s}=1\right)$. 


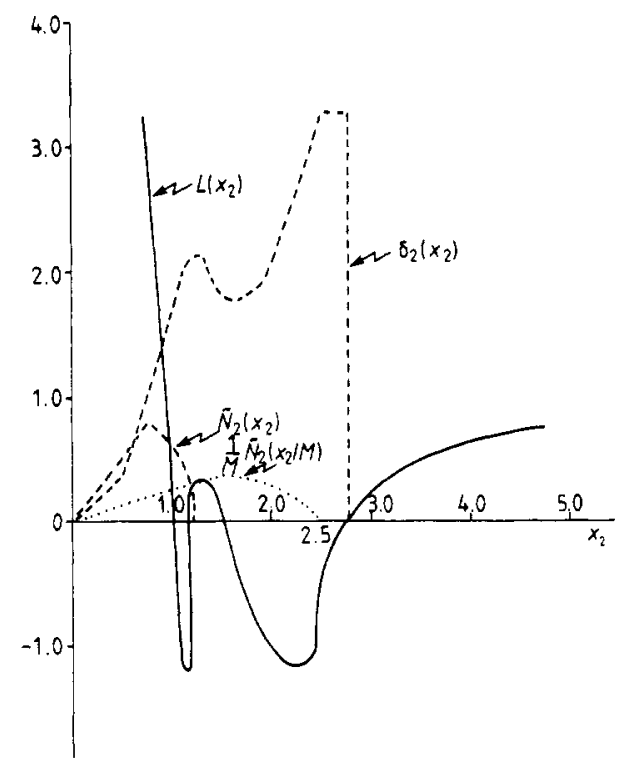

Figure 13. Plot of $\operatorname{Im} L_{+}\left(x_{2}\right)=\left(2 \alpha r_{\mathrm{s}} M / q^{2}\right) \times$ $\left[\tilde{N}_{2}\left(x_{2}\right)+M^{-1} \tilde{N}_{2}\left(x_{2} / M\right)\right], \operatorname{Re} L_{+}\left(x_{2}\right)=L\left(x_{2}\right)$ and the phase angle $\delta_{2}\left(x_{2}\right)$ for mass ratio $M=2, r_{s}=1$ and momentum transfer $q=0.5<q_{2}$, where $x_{2}^{\mathrm{R}}\left(q_{2}\right)=$ $M\left(\frac{1}{2} q_{2}+1\right)$.

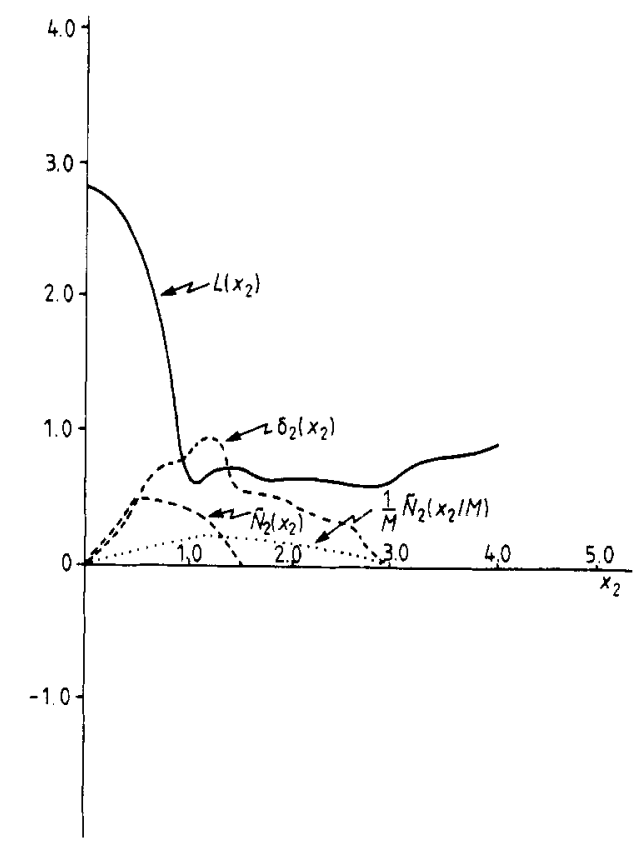

Figure 14. Same as figure 13 but at a momentum transfer $q=1.0>q_{2}$.

(Lahoz 1986). Using our results on the analytic structure of $K\left(z_{1}\right)$ together with Cauchy's theorem, we find by comparison with $G_{1}\left(z_{1}\right) G_{1}\left(-z_{1}\right)=V_{11}^{2} / K\left(z_{1}\right)$ that

$1=\left[1+P\left(z_{1}\right)\right]\left[1+P\left(-z_{1}\right)\right] J_{0}\left(M z_{1}\right) J_{0}\left(-M z_{1}\right)$

$$
\times \begin{cases}1 & q \geqslant q_{1} \\ \frac{\left(1+\frac{1}{2} q+2_{1}\right)\left(1+\frac{1}{2} q-z_{1}\right)}{\left(x_{1}^{\mathrm{R}}+z_{1}\right)\left(x_{1}^{\mathrm{R}}-z_{1}\right)} & q<q_{1} .\end{cases}
$$

With use of the conditions on $P\left(z_{1}\right)$ and the definition of $J_{0}\left(M z_{1}\right)$, equation (4.32) yields (Lahoz 1986)

$$
\left[1+P\left(z_{1}\right)\right] J_{0}\left(M z_{1}\right)=\left\{\begin{array}{ll}
1 & q \geqslant q_{1} \\
\left(x_{1}^{\mathrm{R}}-z_{1}\right) /\left(1+\frac{1}{2} q-z_{1}\right) & q<q_{1}
\end{array} \quad M \text { finite }(\geqslant 1) .\right.
$$

Comparison of (4.19) and (4.33) yields the unique solution

$$
G_{1}\left(z_{1}\right)=\frac{V_{11}}{K\left(z_{1}\right)} \exp \left(\frac{1}{\pi} \int_{\tilde{L}_{1}} \mathrm{~d} x_{1}^{\prime} \frac{\delta_{1}\left(x_{1}^{\prime}\right)}{x_{1}^{\prime}-z_{1}}\right)
$$




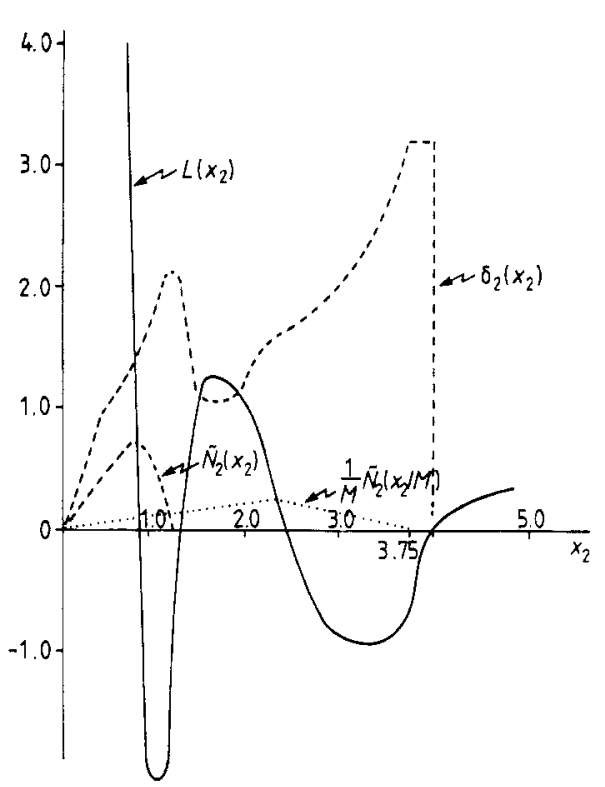

Figure 15. Same as figure 13 but with mass ratio $M=3$.

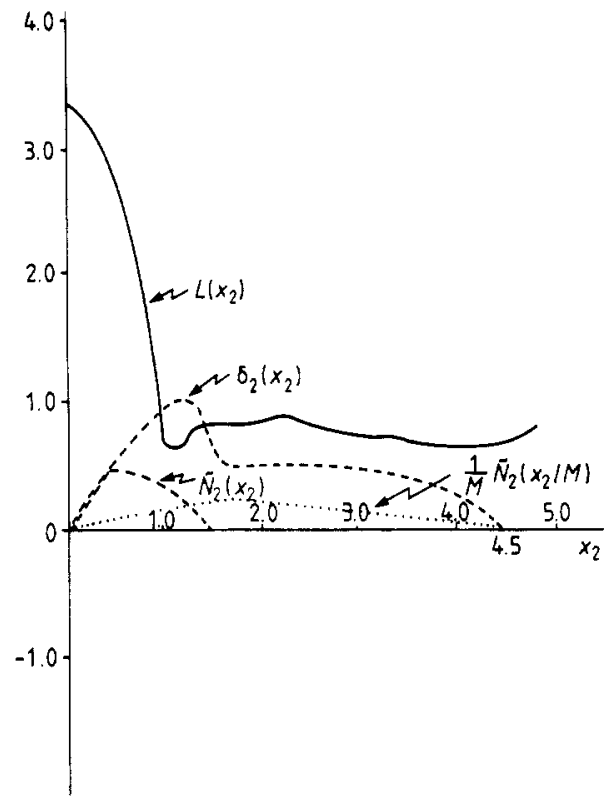

Figure 16. Same as figure 13 but with mass ratio $M=3$ and at momentum transfer $q=1.0>q_{2}$.

$$
\times\left\{\begin{array}{ll}
1 & q \geqslant q_{1} \\
\frac{\left(x_{1}^{\mathrm{R}}-z_{1}\right)}{\left(1+\frac{1}{2} q-z_{1}\right)} & q<q_{1}
\end{array} \quad M \text { finite }(\geqslant 1) .\right.
$$

Similarly, we obtain the unique solution

$$
\begin{aligned}
H_{1}\left(z_{2}\right)=\frac{V_{22}}{L\left(z_{2}\right)} & \exp \left(\frac{1}{\pi} \int_{M \tilde{L}_{2}} \mathrm{~d} x_{2}^{\prime} \frac{\delta_{2}\left(x_{2}^{\prime}\right)}{x_{2}^{\prime}-z_{2}}\right) \\
& \times\left\{\begin{array}{ll}
1 & q \geqslant q_{2} \\
\frac{\left(x_{2}^{\mathrm{R}}-z_{2}\right)}{\left(1+\frac{1}{2} q-z_{2}\right)} & q<q_{2}
\end{array} \quad M \text { finite }(\geqslant 1) .\right.
\end{aligned}
$$

Equivalent forms of (4.34) and (4.35) are readily obtained by analogy to the onecomponent case (Bishop and Lührmann 1978).

As the physical quantities we are interested in, namely the four-point functions $X_{2}, Y_{2}, Z_{2}$ and the correlation energy $E_{\mathrm{c}}$, are expressible in terms of $G_{1}\left(\kappa_{1}\right)$ and $H_{1}\left(\kappa_{2}\right)$ it is not necessary to obtain $g\left(\kappa_{1}\right), \ldots, h\left(\kappa_{2}\right)$. Use of (3.24) yields the expression (where the momenta have been rendered dimensionless)

$$
X_{2 ; \kappa_{1} \kappa_{\mathrm{i}}}(q)=\frac{-4 \alpha r_{\mathrm{s}}}{3 \pi q^{2} N_{1}} \frac{G_{1}\left(\boldsymbol{\kappa}_{1}\right) G_{1}\left(\boldsymbol{\kappa}_{1}^{\prime}\right)}{V_{11}^{2} q \cdot\left(\kappa_{1}^{\prime}+\kappa_{1}\right)}
$$

Similar expressions are obtained for $Y_{2}$ and $Z_{2}$. 
Using equation (3.5) we obtain the following relation for the correlation energy $E_{\mathrm{c}}\left(\equiv E-E_{0}=\left\langle\Phi_{\mathrm{F}}|H| \Psi\right\rangle-\left\langle\Phi_{\mathrm{F}}|H| \Phi_{\mathrm{F}}\right)\right)$ :

$$
\begin{aligned}
E_{c}=\frac{8 \pi e^{2}}{\Omega} \sum_{\boldsymbol{q} \neq 0} & \frac{1}{q^{2}}\left(\sum_{\boldsymbol{\kappa}_{1} \in \tilde{\Gamma}_{1}} \sum_{-\boldsymbol{\kappa}_{1}^{\prime} \in \tilde{\Gamma}_{1}} X_{2 ; \boldsymbol{\kappa}_{1} \boldsymbol{\kappa}_{i}}(\boldsymbol{q})+\sum_{\boldsymbol{\alpha}_{2} \in \tilde{\Gamma}_{2}} \sum_{-\boldsymbol{\kappa}_{2}^{\prime} \in \tilde{\Gamma}_{2}^{\prime}}\right. \\
& \left.\times Y_{2 ; \boldsymbol{\kappa}_{2} \kappa_{i}}(\boldsymbol{q})-2 \sum_{\boldsymbol{\kappa}_{1} \in \tilde{\Gamma}_{1}} \sum_{-\boldsymbol{\kappa}_{2} \in \tilde{\Gamma}_{2}} Z_{2 ; \boldsymbol{\kappa}_{1} \kappa_{2}}(\boldsymbol{q})\right)
\end{aligned}
$$

where we have used $S_{1}=0$ and $e_{1}=-e_{2}=e$.

Using (4.37), $N_{1}=N_{2}$ and $E_{\mathrm{c}}=\varepsilon_{\mathrm{c}} N_{1} e^{2} / 2 a_{1}$ gives

$$
\varepsilon_{\mathrm{c}}^{\mathrm{RPA}}=\frac{3}{8 \pi \alpha r_{\mathrm{s}}}\left\{\int \mathrm{d} \boldsymbol{q} \frac{1}{q^{2}}\left[\int_{\tilde{\Gamma}_{1}} \mathrm{~d} \boldsymbol{\kappa}_{1}\left(\frac{G_{1}\left(\boldsymbol{\kappa}_{1}\right)}{V_{11}}-1\right)+\int_{\tilde{\Gamma}_{2}} \mathrm{~d} \boldsymbol{\kappa}_{2}\left(\frac{H_{1}\left(\boldsymbol{\kappa}_{2}\right)}{V_{22}}-1\right)\right]\right\} .
$$

Equation (4.38) is evaluated by making use of the relations (Lahoz 1986)

$$
\begin{aligned}
& \int_{\tilde{\Gamma}_{1}} \mathrm{~d} \boldsymbol{\kappa}_{1} \frac{G_{1}\left(\boldsymbol{\kappa}_{1}\right)}{V_{11}}+\int_{\tilde{\Gamma}_{2}} \mathrm{~d} \boldsymbol{\kappa}_{2} \frac{H_{1}\left(\boldsymbol{\kappa}_{2}\right)}{V_{22}}=\lim _{z_{1} \rightarrow \infty} 2 \pi \tilde{\beta} q z_{1}\left(1-\frac{G_{1}\left(z_{1}\right) K\left(z_{1}\right)}{V_{11}}\right) \\
& =\lim _{z_{2} \rightarrow \infty} \frac{2 \pi \tilde{\beta} q z_{2}}{M}\left(1-\frac{H_{1}\left(z_{2}\right) L\left(z_{2}\right)}{V_{22}}\right) \\
& \int_{\tilde{\Gamma}_{t}} \mathrm{~d} \kappa_{i}=2 \tilde{\beta} q \int_{\tilde{L}_{1}} \mathrm{~d} x_{i} M^{1-i} \operatorname{Im} K_{+}\left(M^{1-i} x_{i}\right)
\end{aligned}
$$

to give

$$
\begin{aligned}
\varepsilon_{\mathrm{c}}^{\mathrm{RPA}}=\left(\frac{3}{2 \pi}\right)( & \left.\frac{1}{\alpha r_{\mathrm{s}}}\right)^{2} \int_{0}^{\infty} \mathrm{d} q q^{3} \int_{0}^{\infty} \mathrm{d} x_{1}\left[\operatorname{Tan}^{-1}\left(\frac{\operatorname{Im} K_{+}\left(x_{1}\right)}{\operatorname{Re} K_{+}\left(x_{1}\right)}\right)-\operatorname{Im} K_{+}\left(x_{1}\right)\right] \\
& +\frac{1}{M}\left(\frac{3}{2 \pi}\right)\left(\frac{1}{\alpha r_{\mathrm{s}}}\right)^{2} \int_{0}^{\infty} \mathrm{d} q q^{3} \int_{0}^{\infty} \mathrm{d} x_{2}\left[\operatorname{Tan}^{-1}\left(\frac{\operatorname{Im} L_{+}\left(x_{2}\right)}{\operatorname{Re} L_{+}\left(x_{2}\right)}\right)-\operatorname{Im} L_{+}\left(x_{2}\right)\right] \\
= & \left(\frac{3}{2 \pi}\right)\left(\frac{1}{\alpha r_{\mathrm{s}}}\right)^{2} \int_{0}^{\infty} \mathrm{d} q q^{3}\left\{\int_{i_{1}} \mathrm{~d} x_{1}\left[\operatorname{Tan}^{-1}\left(\frac{\operatorname{Im} K_{+}\left(x_{1}\right)}{\operatorname{Re} K_{+}\left(x_{1}\right)}\right)-\operatorname{Im} K_{+}\left(x_{1}\right)\right]\right. \\
& +\pi\left[x_{1}^{\mathrm{R}}-\left(\frac{1}{2} q+1\right)\right] \theta(q 1-q) \\
& +\frac{1}{M} \int_{M L_{2}} \mathrm{~d} x_{2}\left[\operatorname{Tan}^{-1}\left(\frac{\operatorname{Im} L_{+}\left(x_{2}\right)}{\operatorname{Re} L_{+}\left(x_{2}\right)}\right)-\operatorname{Im} L_{+}\left(x_{2}\right)\right] \\
& \left.+\frac{\pi}{M}\left[x_{2}^{\mathrm{R}}-M\left(\frac{1}{2} q+1\right)\right] \theta\left(q_{2}-q\right)\right\}
\end{aligned}
$$

To understand better the constituents of the RPA correlation energy $\varepsilon_{c}^{\text {RPA }}$ we write

$$
\varepsilon_{\mathrm{c}}^{\mathrm{RPA}}=\sum_{i=1,2}\left(\varepsilon_{\mathrm{c} ; \mathrm{cont}, i}^{\mathrm{RPA}}+\varepsilon_{\mathrm{c} ; \mathrm{pl}, i}^{\mathrm{RPA}}\right)
$$

where $\varepsilon_{\mathrm{c} ; \mathrm{cont}, i}^{\mathrm{RPA}}$ refers to the contribution from the integration regime $M^{i-1} \tilde{L}_{i}$ and $\varepsilon_{\mathrm{c} ; \mathrm{p} 1, i}^{\mathrm{RPA}}$ refers to the term involving $x_{i}^{\mathrm{R}}$. Clearly, the term $\varepsilon_{\mathrm{c} ; \mathrm{cont}, i}^{\mathrm{RP}}$ arises from the physical regime $M^{i-1} \tilde{L}_{1}$ corresponding to the particle-hole (p-h) continuum for which $\left|\boldsymbol{\kappa}_{i}-\frac{1}{2} \boldsymbol{q}\right|<$ 1 and $\left|\boldsymbol{\kappa}_{i}+\frac{1}{2} \boldsymbol{q}\right|>1$ and includes the overlap between the integration regions for particles of type $i$ and $j(i \neq j)$; and the term $\varepsilon_{\mathrm{c} ; \mathrm{pl}, i}^{\mathrm{RPA}}$ arises from an unphysical (for bare $\mathrm{p}-\mathrm{h}$ pairs) region in momentum space. 
In the limit $M \rightarrow \infty$, when the heavier particles become a rigid background to the lighter ones, we expect the former not to vibrate and possess no phonon. When $M$ is close to unity we do not expect such screening to take place so a plasmon rather than a phonon will be observed. By means of an analysis similar to that in the one-component case (Bishop and Lührmann 1978) one sees that $x_{i}^{\mathrm{R}}=x_{i}^{\mathrm{R}}(q)$ essentially measures the plasmon dispersion curve for $i=1$ and the plasmon or phonon dispersion curve for $i=2$. With this physical interpretation of the zero $z_{i}=x_{i}^{\mathrm{R}}$, the fact that $L\left(z_{2}\right)$ has no zero in the limit $M \rightarrow \infty$ is understood immediately, for the particles described by $L\left(z_{2}\right)$ will not vibrate and consequently possess no phonon (zero).

By analogy with ordinary two-body potential scattering we expect the cut $M^{i-1} \tilde{L_{i}}$ of the three-point function to be a reflection of the $\mathrm{p}-\mathrm{h}$ continuum for species $i$ and the (possible) pole $z_{i}=x_{i}^{R}$ of the same function to correspond to a bound state, which is a true (many $p-h$ ) collective excitation of the many-body system. We show in $\$ 7$ that the above interpretation is correct.

Consider the following cases of interest: (i) $M=1$, which provides a first approximation to an electron-hole (e-h) system with an excitonic phase (Knox 1963), (ii) $M=1836$, which provides a description of a hydrogen plasma; and (iii) the limit $M \rightarrow \infty$.

It is straightforward to show (Lahoz 1986) that for $M=1$ :

$$
K^{\mathrm{e}-\mathrm{h}}\left(x_{1}\right)=L^{\mathrm{e}-\mathrm{h}}\left(x_{1}\right)=1+\frac{2 \alpha r_{\mathrm{s}}}{\pi q^{3}} K^{(1)}\left(x_{1}\right)
$$

and $q_{1}=q_{2}=q_{\max }$ is given by

$$
q_{\max }^{2}=\frac{2 \alpha r_{\mathrm{s}}}{\pi}\left\{\left[q_{\max }+2\right] \ln \left(1+2 / q_{\max }\right)-2\right\}
$$

which is seen to have a unique solution for all physical $r_{\mathrm{s}}$. For $r_{\mathrm{s}}=1$, for example, it has solution $q_{\max }=0.729$. The structure of the real and imaginary parts of $K_{+}^{\mathrm{e}-\mathrm{h}}\left(x_{1}\right)$ together with the phase angle $\delta_{1}^{\text {e-h }}\left(x_{1}\right)$ is highlighted in figures 6 and 7 for the cases $q>q_{\max }$ and $q<q_{\max }$ for $q<2$. Nothing of significance changes for $q>2$.

It is easy to show that

$$
\begin{aligned}
& G_{1}^{e-h}\left(z_{1}\right)=H_{1}^{e-h}\left(z_{1}\right)=\frac{V_{11}}{K^{e-h}\left(x_{1}\right)} \exp \left(\frac{1}{\pi} \int_{\tilde{L}_{\mathrm{t}}} \mathrm{d} x_{1}^{\prime} \frac{\delta^{\mathrm{e}-\mathrm{h}}\left(x_{1}^{\prime}\right)}{x_{1}^{\prime}-z_{1}}\right) \\
& \times \begin{cases}1 & q \geqslant q_{\max } \\
\frac{\left(x_{1}^{\mathrm{R}}-z_{1}\right)}{\left(1+\frac{1}{2} q-z_{1}\right)} & q<q_{\max }\end{cases} \\
& \varepsilon_{\mathrm{c}}^{\mathrm{RPA} ; \mathrm{e}-\mathrm{h}}=\left(\frac{3}{2 \pi}\right)\left(\frac{1}{\alpha r_{\mathrm{s}}}\right)^{2} \int_{0}^{\infty} \mathrm{d} q q^{3} \\
& \times \sum_{i=1,2}\left\{\int _ { \tilde { L } _ { i } } \mathrm { d } x _ { i } \left[\operatorname{Tan}^{-1}\left(\frac{\operatorname{Im} K_{+}^{\mathrm{e}-\mathrm{h}}\left(x_{i}\right)}{\operatorname{Re} K_{+}^{\mathrm{e}-\mathrm{h}}\left(x_{i}\right)}\right)\right.\right. \\
& \left.\left.-\operatorname{Im} K_{+}^{\mathrm{e}-\mathrm{h}}\left(x_{i}\right)\right]+\pi\left[x_{i}^{\mathrm{R}}-\left(\frac{1}{2} q+1\right)\right] \theta\left(q_{\max }-q\right)\right\}
\end{aligned}
$$

where $x_{i}^{\mathrm{R}}=x_{i}^{\mathrm{R}}(q)$ is as previously discussed. The equality of the three-point functions for each type of particle is in agreement with Chakraborty and Pietiläinen (1982), who use a variational approach. 
It can be shown (Lahoz 1986) that for large $M, K\left(x_{1}\right)$ has the following behaviour:

$$
K\left(x_{1}\right) \approx \begin{cases}1+\frac{\alpha r_{\mathrm{s}}}{\pi q^{3}} M K^{(1)}\left(M x_{1}\right) & 0<x_{1}<\mathrm{O}\left(M^{-\delta}\right) \\ 1+\frac{\alpha r_{\mathrm{s}}}{\pi q^{3}} K^{(1)}\left(x_{1}\right) & x_{1}>\mathrm{O}\left(M^{-\delta}\right) .\end{cases}
$$

Clearly, by choosing $M$ sufficiently large we can make the dielectric function for the lighter particles $K\left(z_{1}\right)$ be as close as we wish to the one-component dielectric function $K_{\mathrm{R}}\left(z_{1}\right)$, so that in the limit $M \rightarrow \infty K\left(z_{1}\right)$ is equal to $K_{\mathrm{R}}\left(z_{1}\right)$ for $\left|\operatorname{Re} z_{1}\right|>0$; further, one can show that in the same limit $G_{1}\left(z_{1}\right) / V_{11}$ becomes the one-component three-point vertex function $g_{\mathrm{R}}\left(z_{1}\right)$ for $\left|\operatorname{Re} z_{1}\right|>0$. Physically, this result means that replacing the rigid background of the one-component case by a collection of heavy (but not rigid) particles has little effect; in particular this is the case when replacing the rigid background to an electron fluid by a proton fluid to obtain a zero-temperature hydrogen plasma, a result in agreement with Chakraborty et al (1983) who use a variational approach.

Clearly, as $M$ is increased the two-component system approaches the onecomponent case as the heavier particles become more rigid. This gradual change to a system with ordered heavy particles is supported by the behaviour of $L\left(x_{2}\right)$ for large $M$ (Lahoz 1986), and in agreement with Chakraborty et al (1983). In the limit $M \rightarrow \infty$ the heavier particles become a rigid background to the lighter ones and we recover the one-component result as expected.

The possible existence of the liquid metallic hydrogen phase at $T=0$ is of considerable interest. This is likely to occur in a region where the parameter $r_{\mathrm{s}}$ is sufficiently large that our analytic solution has to be treated with care.

\section{RPA treatment of the non-Coulombic potential case}

Here $V_{11} V_{22} \neq V_{12}^{2}$ so the matrix of the potentials $V$ is non-singular and the original three-point functions $g\left(\kappa_{1}\right), \ldots, h\left(\kappa_{2}\right)$ can be obtained directly from the new threepoint functions $G_{1}\left(\kappa_{1}\right), \ldots, H_{2}\left(\kappa_{2}\right)$. The non-Coulombic case does not simplify as the Coulombic one does and consequently, although the techniques are essentially the same, the former requires much greater mathematical manipulation to obtain a general solution for the three-point functions $G_{1}\left(\kappa_{1}\right), \ldots, H_{2}\left(\kappa_{2}\right)$.

It is easy to verify that, as expected, in the limit $V_{12} \rightarrow 0$, the two-component equations decouple into two one-component systems, each neutralised by a suitable inert background.

Before proceeding with the solution of equations (3.26)-(3.29) we remark on the case $q=0$. In this case, for arbitrary potentials $V_{11}, V_{22}, V_{12}$, the LHS of (3.18) is zero, so that an analysis involving energy denominators is not possible. The limit $q \rightarrow 0$, on the other hand, may be well defined. Consequently, we omit the case $q=0$ in our analysis, whilst allowing the possibility of taking the above limit. It will be seen in paper II that the case $q=0$ is intimately connected with superconductivity.

We linearise (3.26)-(3.29) by using a generalisation of the trick employed in the one-component case. These manipulations yield the equations

$$
G_{1}\left(\boldsymbol{\kappa}_{1}\right) W\left(\boldsymbol{\kappa}_{1}\right)=1+\frac{1}{2 \pi \beta_{0}}\left(\int_{\Gamma_{1}^{\prime}} \mathrm{d} \boldsymbol{\kappa}_{1}^{\prime} \frac{G_{1}\left(\boldsymbol{\kappa}_{1}^{\prime}\right)}{q \cdot\left(\boldsymbol{\kappa}_{1}^{\prime}-\boldsymbol{\kappa}_{1}\right)}+\frac{V_{12}}{T\left(\boldsymbol{\kappa}_{1}\right)} \int_{\Gamma_{2}^{\prime}} \mathrm{d} \boldsymbol{\kappa}_{2}^{\prime} \frac{M H_{2}\left(\boldsymbol{\kappa}_{2}^{\prime}\right)}{q \cdot\left(\boldsymbol{\kappa}_{2}^{\prime}-M \boldsymbol{\kappa}_{1}\right)}\right)
$$




$$
\begin{aligned}
H_{2}\left(\boldsymbol{\kappa}_{2}\right) \hat{W}\left(\boldsymbol{\kappa}_{2}\right) & =\frac{1}{2 \pi \beta_{0}} \int_{\Gamma_{2}^{\prime}} \mathrm{d} \boldsymbol{\kappa}_{2}^{\prime} \frac{M H_{2}\left(\boldsymbol{\kappa}_{2}^{\prime}\right)}{q \cdot\left(\boldsymbol{\kappa}_{2}^{\prime}-\boldsymbol{\kappa}_{2}\right)} \\
& +\frac{V_{12}}{U\left(\boldsymbol{\kappa}_{2}\right)}\left(1+\frac{1}{2 \pi \beta_{0}} \int_{\Gamma_{1}^{\prime}} \mathrm{d} \boldsymbol{\kappa}_{1}^{\prime} \frac{G_{1}\left(\boldsymbol{\kappa}_{1}^{\prime}\right)}{q \cdot\left(\boldsymbol{\kappa}_{1}^{\prime}-\boldsymbol{\kappa}_{2} / M\right)}\right) \\
G_{2}\left(\boldsymbol{\kappa}_{1}\right) W\left(\boldsymbol{\kappa}_{1}\right) & =\frac{1}{2 \pi \beta_{0}} \int_{\Gamma_{1}^{\prime}} \mathrm{d} \boldsymbol{\kappa}_{1}^{\prime} \frac{G_{2}\left(\boldsymbol{\kappa}_{1}^{\prime}\right)}{q \cdot\left(\boldsymbol{\kappa}_{1}^{\prime}-\boldsymbol{\kappa}_{1}\right)} \\
& +\frac{V_{12}}{T\left(\boldsymbol{\kappa}_{1}\right)}\left(1+\frac{1}{2 \pi \beta_{0}} \int_{\Gamma_{2}^{\prime}} \mathrm{d} \boldsymbol{\kappa}_{2}^{\prime} \frac{M H_{1}\left(\boldsymbol{\kappa}_{2}^{\prime}\right)}{\boldsymbol{q} \cdot\left(\boldsymbol{\kappa}_{2}^{\prime}-M \boldsymbol{\kappa}_{1}\right)}\right) \\
H_{1}\left(\boldsymbol{\kappa}_{2}\right) \hat{W}\left(\boldsymbol{\kappa}_{2}\right) & =1+\frac{1}{2 \pi \beta_{0}}\left(\int_{\Gamma_{2}^{\prime}} \mathrm{d} \boldsymbol{\kappa}_{2}^{\prime} \frac{M H_{1}\left(\boldsymbol{\kappa}_{2}^{\prime}\right)}{\boldsymbol{q} \cdot\left(\boldsymbol{\kappa}_{2}^{\prime}-\boldsymbol{\kappa}_{2}\right)}\right. \\
& \left.+\frac{V_{12}}{U\left(\boldsymbol{\kappa}_{2}\right)} \int_{\Gamma_{1}^{\prime}} \mathrm{d} \boldsymbol{\kappa}_{1}^{\prime} \frac{G_{2}\left(\boldsymbol{\kappa}_{1}^{\prime}\right)}{q \cdot\left(\boldsymbol{\kappa}_{1}^{\prime}-\boldsymbol{\kappa}_{2} / M\right)}\right)
\end{aligned}
$$

where we have defined

$$
\begin{aligned}
& \mathbf{W}\left(\boldsymbol{\kappa}_{1}\right) \equiv\left[\begin{array}{cc}
W_{0}\left(\boldsymbol{\kappa}_{1}\right) & W_{1}\left(\boldsymbol{\kappa}_{1}\right) \\
W_{2}\left(M \boldsymbol{\kappa}_{1}\right) & W_{3}\left(M \boldsymbol{\kappa}_{1}\right)
\end{array}\right] \\
& =\left[\begin{array}{ll}
1 & 0 \\
0 & 1
\end{array}\right]+\frac{1}{2 \pi \beta_{0}}\left[\begin{array}{c:c:c}
\int_{\Gamma_{1}^{\prime}} \mathrm{d} \boldsymbol{\kappa}_{1}^{\prime}\left(\frac{1}{q \cdot\left(\boldsymbol{\kappa}_{1}^{\prime}+\boldsymbol{\kappa}_{1}\right)}+\frac{1}{q \cdot\left(\boldsymbol{\kappa}_{1}^{\prime}+\boldsymbol{\kappa}_{1}\right)}\right) & 0 \\
\hdashline 0 & \int_{\Gamma_{2}^{\prime}} \mathrm{d} \boldsymbol{\kappa}_{2}^{\prime} M\left(\frac{1}{q \cdot\left(\boldsymbol{\kappa}_{2}^{\prime}+M \boldsymbol{\kappa}_{1}\right)}+\frac{1}{q \cdot\left(\boldsymbol{\kappa}_{2}^{\prime}-M \boldsymbol{\kappa}_{1}\right)}\right)
\end{array}\right] \\
& \times\left[\begin{array}{ll}
V_{11} & V_{12} \\
V_{12} & V_{22}
\end{array}\right] \\
& W\left(\kappa_{1}\right) \equiv \operatorname{det}\left[W\left(\kappa_{1}\right)\right] / T\left(\kappa_{1}\right) \quad T\left(\kappa_{1}\right) \equiv V_{11} W_{3}\left(M \kappa_{1}\right)-V_{12} W_{2}\left(M \kappa_{1}\right) \\
& \hat{W}\left(\kappa_{2}\right) \equiv \operatorname{det}\left[\mathbf{W}\left(\kappa_{2} / M\right)\right] / U\left(\boldsymbol{\kappa}_{2}\right) \quad U\left(\kappa_{2}\right) \equiv V_{22} W_{0}\left(\kappa_{2} / M\right)-V_{12} W_{1}\left(\kappa_{2} / M\right) .
\end{aligned}
$$

To decouple (5.1)-(5.4) we introduce, for functions $I_{0}^{-1}\left(\kappa_{1}\right), J_{0}^{-1}\left(\kappa_{2}\right)$, in principle solvable linear integral equations and use the same trick as in the Coulombic case to obtain

$$
G_{1}\left(\boldsymbol{\kappa}_{1}\right) F\left(\boldsymbol{\kappa}_{1}\right)=1+\frac{1}{2 \pi \beta_{0}} \int_{\Gamma_{i}^{\prime}} \mathrm{d} \boldsymbol{\kappa}_{1}^{\prime} \frac{G_{1}\left(\boldsymbol{\kappa}_{1}^{\prime}\right)}{q \cdot\left(\boldsymbol{\kappa}_{1}^{\prime}-\boldsymbol{\kappa}_{1}\right)}+\frac{J\left(\boldsymbol{\kappa}_{1}\right)}{2 \pi \beta_{0}} \int_{\Gamma_{1}^{\prime}} \mathrm{d} \boldsymbol{\kappa}_{1}^{\prime} \frac{G_{1}\left(\boldsymbol{\kappa}_{1}^{\prime}\right) \hat{V}\left(\boldsymbol{\kappa}_{1}^{\prime}\right)}{q \cdot\left(\boldsymbol{\kappa}_{1}^{\prime}-\boldsymbol{\kappa}_{1}\right)}
$$

where we have defined

$$
\begin{aligned}
F\left(\boldsymbol{\kappa}_{1}\right) & \equiv \frac{\operatorname{det}\left[\mathbf{W}\left(\boldsymbol{\kappa}_{1}\right)\right]}{\left[T\left(\boldsymbol{\kappa}_{1}\right)+\left(V_{12} / 2 \pi \beta_{0}\right) \hat{V}\left(\boldsymbol{\kappa}_{1}\right) J_{0}\left(M \boldsymbol{\kappa}_{1}\right)\right]} \\
J\left(\boldsymbol{\kappa}_{1}\right) & \equiv \frac{-V_{12}}{2 \pi \beta_{0}} \frac{J_{0}\left(M \boldsymbol{\kappa}_{1}\right) F\left(\boldsymbol{\kappa}_{1}\right)}{\operatorname{det}\left[\mathbf{W}\left(\boldsymbol{\kappa}_{1}\right)\right]} \\
\hat{V}\left(\boldsymbol{\kappa}_{1}\right) & \equiv \int_{\Gamma_{2}^{\prime}} \mathrm{d} \boldsymbol{\kappa}_{2}^{\prime} \frac{M V_{12}}{q \cdot\left(\boldsymbol{\kappa}_{2}^{\prime}-M \boldsymbol{\kappa}_{1}\right) \hat{W}\left(\boldsymbol{\kappa}_{2}^{\prime}\right) J_{0}\left(\boldsymbol{\kappa}_{2}^{\prime}\right) U\left(\boldsymbol{\kappa}_{2}^{\prime}\right)}
\end{aligned}
$$


and

$$
\begin{aligned}
H_{2}\left(\boldsymbol{\kappa}_{2}\right) \hat{F}\left(\boldsymbol{\kappa}_{2}\right)= & -2 \pi \beta_{0} I\left(\boldsymbol{\kappa}_{2}\right)+\frac{1}{2 \pi \beta_{0}} \int_{\Gamma_{2}^{\prime}} \mathrm{d} \boldsymbol{\kappa}_{2}^{\prime} \frac{M H_{2}\left(\boldsymbol{\kappa}_{2}^{\prime}\right)}{q \cdot\left(\boldsymbol{\kappa}_{2}^{\prime}-\boldsymbol{\kappa}_{2}\right)} \\
& +\frac{I\left(\boldsymbol{\kappa}_{2}\right)}{2 \pi \beta_{0}} \int_{\Gamma_{2}^{\prime}} \mathrm{d} \boldsymbol{\kappa}_{2}^{\prime} \frac{M H_{2}\left(\boldsymbol{\kappa}_{2}^{\prime}\right) \hat{Y}\left(\boldsymbol{\kappa}_{2}^{\prime}\right)}{q \cdot\left(\boldsymbol{\kappa}_{2}^{\prime}-\boldsymbol{\kappa}_{2}\right)}
\end{aligned}
$$

where we have defined

$$
\begin{aligned}
\hat{F}\left(\kappa_{2}\right) & \equiv \frac{\operatorname{det}\left[\mathbf{W}\left(\boldsymbol{\kappa}_{2} / M\right)\right]}{\left[U\left(\boldsymbol{\kappa}_{2}\right)+\left(V_{12} / 2 \pi \beta_{0}\right) \hat{Y}\left(\boldsymbol{\kappa}_{2}\right) I_{0}\left(\boldsymbol{\kappa}_{2} / M\right)\right]} \\
I\left(\boldsymbol{\kappa}_{2}\right) & \equiv \frac{-V_{12}}{2 \pi \beta_{0}} \frac{I_{0}\left(\boldsymbol{\kappa}_{2} / M\right) \hat{F}\left(\boldsymbol{\kappa}_{2}\right)}{\operatorname{det}\left[\mathbf{W}\left(\boldsymbol{\kappa}_{2} / M\right)\right]} \\
\hat{Y}\left(\boldsymbol{\kappa}_{2}\right) & \equiv \int_{\Gamma_{1}} \operatorname{d} \boldsymbol{\kappa}_{1}^{\prime} \frac{V_{12}}{q \cdot\left(\boldsymbol{\kappa}_{1}^{\prime}-\boldsymbol{\kappa}_{2} / M\right) W\left(\boldsymbol{\kappa}_{1}^{\prime}\right) I_{0}\left(\boldsymbol{\kappa}_{1}^{\prime}\right) T\left(\boldsymbol{\kappa}_{1}^{\prime}\right)} .
\end{aligned}
$$

Similar equations are obtained for $G_{2}\left(\kappa_{1}\right), H_{1}\left(\kappa_{2}\right)$.

To complete the analogy between the non-Coulombic and Coulombic cases we require functional relations involving the dielectric-like functions $F\left(\kappa_{1}\right), \hat{F}\left(\kappa_{2}\right)$. It can be shown (Lahoz 1986) that the following relation holds:

$$
\begin{aligned}
& \operatorname{det}\left[\mathbf{W}\left(\boldsymbol{\kappa}_{1}\right)\right]=\frac{(\operatorname{det} \mathbf{V})^{2}}{\operatorname{det}\left[\mathbf{H}\left(\boldsymbol{\kappa}_{1}\right)\right] \operatorname{det}\left[\mathbf{H}\left(-\boldsymbol{\kappa}_{1}\right)\right]} \\
& \mathbf{H}\left(\boldsymbol{\kappa}_{1}\right) \equiv\left[\begin{array}{cc}
G_{1}\left(\boldsymbol{\kappa}_{1}\right) & G_{2}\left(\boldsymbol{\kappa}_{1}\right) \\
H_{2}\left(\boldsymbol{M} \boldsymbol{\kappa}_{1}\right) & H_{1}\left(\boldsymbol{M} \boldsymbol{\kappa}_{1}\right)
\end{array}\right]
\end{aligned}
$$

where the matrices $\mathbf{W}, \mathbf{V}$ have already been defined. From equation (5.9) and the definitions of $F\left(\boldsymbol{\kappa}_{1}\right)$ and $\hat{F}\left(\boldsymbol{\kappa}_{2}\right)$ functional relations involving these are readily obtained. Clearly, equation (5.9) is the two-component generalisation of the one-component result.

The method employed to solve the above linear equations is essentially the same as used in the Coulombic case. General solutions under certain assumptions have been obtained by Lahoz (1986).

The non-Coulombic case RPA equations obtained in this section are suitable for studying two-component systems such as metals where the electron-electron and ion-ion potentials are Coulombic in nature, and the electron-ion potential found from solid state theory.

\section{Excited-state one- and two-body equations}

Consider a two-compartment fermion system with Hamiltonian given by equation (3.1). The Schrödinger equation in the excited-state formalism is given by:

$$
H\left|\Psi_{\mathrm{e}}\right\rangle=E_{\mathrm{e}}\left|\Psi_{\mathrm{e}}\right\rangle
$$

Projecting equation (6.1) from the left with the $n \mathrm{p}-n \mathrm{~h}$ states built from the filled Fermi sea, we obtain the system of equations

$$
\left\langle\Phi_{\mathrm{F}}\left|a_{\lambda_{1}}^{\dagger} \ldots a_{\lambda_{n}}^{\dagger} a_{\eta_{n}} \ldots a_{\eta_{1}} H\right| \Psi_{e}\right\rangle=E_{e}\left\langle\eta_{1} \ldots \eta_{n}\left|\Psi_{n}^{e}\right| \lambda_{1} \ldots \lambda_{n}\right\rangle_{\mathrm{A}}
$$


which is equivalent to a system of coupled equations for the amplitudes $\Psi_{n}^{e}$ defined as

$$
\left\langle\eta_{1} \ldots \eta_{n}\left|\Psi_{n}^{\mathrm{e}}\right| \lambda_{1} \ldots \lambda_{n}\right\rangle_{\mathrm{A}}=\left\langle\Phi_{\mathrm{F}}\left|a_{\lambda_{1}}^{\dagger} \ldots a_{\lambda_{n}}^{\dagger} a_{\eta_{n}} \ldots a_{\eta_{1}}\right| \Psi_{\mathrm{e}}\right\rangle
$$

where we have adopted the same nomenclature as in the two-component ground-state formalism.

Use of the Hamiltonian under consideration and the relation $0=\left\langle\Phi_{F}|H| \Psi_{e}\right\rangle$ yields

$$
\begin{aligned}
0=\sum_{\nu}\left\langle\nu\left|T_{1} \Psi_{1}^{\mathrm{e}}\right| \nu\right\rangle & +\sum_{\mu}\left\langle\mu\left|T_{2} \Psi_{1}^{\mathrm{e}}\right| \mu\right\rangle+\frac{1}{2} \sum_{\nu \nu^{\prime}}\left\langle\nu \nu^{\prime}\left|V_{11} \Psi_{2}^{\mathrm{e}}\right| \nu \nu^{\prime}\right\rangle_{\mathrm{A}} \\
& +\frac{1}{2} \sum_{\mu \mu^{\prime}}\left\langle\mu \mu^{\prime}\left|V_{22} \Psi_{2}^{\mathrm{e}}\right| \mu \mu^{\prime}\right\rangle_{\mathrm{A}}+\sum_{\nu \mu}\left\langle\nu \mu\left|V_{12} \Psi_{2}^{\mathrm{e}}\right| \nu \mu\right\rangle .
\end{aligned}
$$

Following Emrich (1981a, b) we now make the ansatz

$$
\left|\Psi_{\mathrm{e}}\right\rangle=S^{\mathrm{e}}\left|\Psi_{\mathrm{g}}\right\rangle=S^{\mathrm{e}} \exp \left(S^{\mathrm{g}}\right)\left|\Phi_{\mathrm{F}}\right\rangle
$$

where the g subscript/superscript corresponds to the ground-state quantity, and $S^{e}$, $S_{n}^{e}$ are given by $(3.8 b)$ and $(3.8 c)$ with an e superscript. As in the ground state $\left\langle\ldots \lambda \ldots \mid S_{n}^{\mathrm{e}} \ldots\right\rangle=\left\langle\ldots\left|S_{n}^{\mathrm{e}}\right| \ldots \eta \ldots\right\rangle=0$.

Insertion of equations (6.5) in equation (6.3) gives the decompositions

$$
\left\langle\eta\left|\Psi_{1}^{\mathbf{e}}\right| \lambda\right\rangle=\sum_{\theta}\langle\eta \mid \theta\rangle\left\langle\theta\left|S_{\mathbf{i}}^{\mathbf{e}}\right| \lambda\right\rangle
$$

$\left\langle\eta_{1} \eta_{2}\left|\Psi_{2}^{\mathrm{e}}\right| \lambda_{1} \lambda_{2}\right\rangle=\sum_{(\mathrm{g}, \mathrm{e})}\left[\left\langle\eta_{1}\left|\Psi_{1}^{\mathrm{g}}\right| \lambda_{1}\right\rangle\left\langle\eta_{2}\left|\Psi_{1}^{\mathrm{e}}\right| \lambda_{2}\right\rangle\right]+\left\langle\eta_{1} \eta_{2}\left|S_{2}^{\mathrm{e}}\right| \lambda_{1} \lambda_{2}\right\rangle$

$\left\langle\eta_{1} \eta_{2} \eta_{3}\left|\Psi_{3}^{\mathrm{e}}\right| \lambda_{1} \lambda_{2} \lambda_{3}\right\rangle=\sum_{(\mathrm{g}, \mathrm{e})}\left[S_{123}\left\langle\eta_{1}\left|\Psi_{1}^{\mathrm{g}}\right| \lambda_{1}\right\rangle\left\langle\eta_{2} \eta_{3}\left|S_{2}^{\mathrm{e}}\right| \lambda_{2} \lambda_{3}\right\rangle\right]$

$$
+\left\langle\eta_{1} \eta_{2} \eta_{3}\left|S_{3}^{\mathrm{e}}\right| \lambda_{1} \lambda_{2} \lambda_{3}\right\rangle
$$

where we have defined

$$
\sum_{(\mathbf{g}, \mathrm{e})} \hat{O}^{\mathrm{g}} \hat{P}^{\mathrm{e}} \equiv \hat{O}^{\mathrm{g}} \hat{P}^{\mathrm{e}}+\hat{O}^{\mathrm{e}} \hat{P}^{\mathrm{g}}
$$

To obtain the exact one- and two-body equations we evaluate

$$
\left\langle\Phi_{\mathrm{F}}\left|a_{\lambda_{1}}^{+} \ldots a_{\lambda_{n}}^{\dagger} a_{\eta_{n}} \ldots a_{\eta_{1}} H\right| \Psi_{\mathrm{e}}\right\rangle=E_{\mathrm{e}}\left\langle\eta_{1} \ldots \eta_{n}\left|\Psi_{n}^{\mathrm{e}}\right| \lambda_{1} \ldots \lambda_{n}\right\rangle_{\mathrm{A}}
$$

for $n=1,2$. The procedure by which this is done is detailed in Lahoz (1986). The one-body equation is

$$
\begin{aligned}
\sum_{i=1}^{2}\left[\left\langle\gamma_{i}\left|T_{i} \Psi_{1}^{\mathrm{e}}\right|\right.\right. & \left.\lambda_{i}\right\rangle+\sum_{j=1}^{2} \sum_{\lambda_{j}}\left(\left\langle\gamma_{i} \lambda_{j}^{\prime}\left|T_{j}(2) S_{2}^{\mathrm{e}}+V_{i j} \Psi_{2}^{\mathrm{e}}\right| \lambda_{i} \lambda_{j}^{\prime}\right\rangle_{\mathrm{A}}\right. \\
& \left.-\sum_{(\mathrm{g}, \mathrm{e})} \delta_{i j}\left\langle\gamma_{i}\left|\Psi_{1}^{\mathrm{g}}\right| \lambda_{j}^{\prime}\right\rangle h_{\lambda_{j}^{\prime} \lambda_{i}}^{(\mathrm{e})}\right)+\frac{1}{2} \sum_{j=1}^{2} \sum_{k=j}^{2} \sum_{\lambda_{j}^{\prime} \lambda_{k}^{\prime \prime}} \\
& \times\left\langle\gamma_{i} \lambda_{j}^{\prime} \lambda_{k}^{\prime \prime}\left|\left(2-\delta_{j k}\right) V_{j k}(23)^{\mathrm{e}} \chi_{3}^{(23)}\right| \lambda_{i} \lambda_{j}^{\prime} \lambda_{k}^{\prime \prime}\right\rangle_{\mathrm{A}} \\
& \left.-\left(E_{\mathrm{e}}-E_{\mathrm{g}}\right)\left\langle\gamma_{i}\left|\Psi_{1}^{\mathrm{e}}\right| \lambda_{i}\right\rangle\right]=0
\end{aligned}
$$

where we have defined

$$
h_{\lambda_{i} \lambda_{i}^{(\mathrm{e})}}^{(\mathrm{g}) /\left(\lambda_{i}\right.} \equiv\left\langle T_{i} \Psi_{1}^{\mathrm{g} / \mathrm{e}} \mid \lambda_{i}^{\prime}\right\rangle+\sum_{\lambda_{i}^{\prime}}\left\langle\lambda_{i} \lambda_{i}^{\prime \prime}\left|V_{i i} \Psi_{2}^{\mathrm{g} / \mathrm{e}}\right| \lambda_{i}^{\prime} \lambda_{i}^{\prime \prime}\right\rangle_{\mathrm{A}}+\sum_{\substack{\lambda_{j}^{\prime \prime} \\ j \neq i}}\left\langle\lambda_{i} \lambda_{j}^{\prime \prime}\left|V_{i j} \Psi_{2}^{\mathrm{g} / \mathrm{e}}\right| \lambda_{i}^{\prime} \lambda_{j}^{\prime \prime}\right\rangle
$$


$\left\langle\left.\gamma_{1} \gamma_{2} \gamma_{3}\right|^{\mathrm{e}} \chi_{3}^{(23)} \mid \lambda_{1} \lambda_{2} \lambda_{3}\right\rangle \equiv S_{23}\left(\sum_{(\mathrm{g}, \mathrm{e})}\left\langle\gamma_{2}\left|\Psi_{1}^{\mathrm{e}}\right| \lambda_{2}\right\rangle\left\langle\gamma_{1} \gamma_{3}\left|S_{2}^{\mathrm{g}}\right| \lambda_{1} \lambda_{3}\right\rangle\right)+\left\langle\gamma_{1} \gamma_{2} \gamma_{3}\left|S_{3}^{\mathrm{e}}\right| \lambda_{1} \lambda_{2} \lambda_{3}\right\rangle$

and $S_{23}$ is the permutation operator introduced in equation (3.9).

The two-body equation is

$$
\begin{aligned}
\sum_{i=1}^{2} \sum_{j=i}^{2}\left[\sum_{(\mathrm{g}, \mathrm{e})}(\right. & \left\langle\gamma_{i} \gamma_{j}^{\prime}\left|\left[Q^{(\mathrm{g})}(1) T_{i}(1)+Q^{(\mathrm{g})}(2) T_{j}(2)\right] S_{2}^{\mathrm{e}}\right| \lambda_{i} \lambda_{j}^{\prime}\right\rangle_{\mathrm{A}} \\
& +\sum_{k=1}^{2} \sum_{\lambda_{k}^{\prime \prime}}\left[\delta_{i k} h_{\lambda_{k}^{\prime} \lambda_{i}}^{(\mathrm{g})}\left\langle\gamma_{i} \gamma_{j}^{\prime}\left|S_{2}^{\mathrm{e}}\right| \lambda_{k}^{\prime \prime} \lambda_{j}^{\prime}\right\rangle_{\mathrm{A}}+\delta_{j k} h_{\lambda_{k}^{\prime} \lambda_{j}^{\prime}}^{(\mathrm{g})}\left\langle\gamma_{i} \gamma_{j}^{\prime}\left|S_{2}^{\mathrm{e}}\right| \lambda_{i} \lambda_{k}^{\prime \prime}\right\rangle_{\mathrm{A}}\right] \\
& +\frac{1}{2} \sum_{\lambda_{i}^{\prime \prime} \lambda_{j}^{\prime \prime}}\left\langle\gamma_{i} \gamma_{j}^{\prime}\left|S_{2}^{\mathrm{e}}\right| \lambda_{i}^{\prime \prime} \lambda_{j}^{\prime \prime \prime}\right\rangle_{\mathrm{A}}\left\langle\lambda_{i}^{\prime \prime} \lambda_{j}^{\prime \prime \prime}\left|\left(2-\delta_{i j}\right) V_{i j}\right| \lambda_{i} \lambda_{j}^{\prime}\right\rangle_{\mathrm{A}}+\sum_{k=1}^{2} \sum_{\lambda_{k}^{\prime \prime}}\left\langle\gamma_{i} \gamma_{j}^{\prime} \lambda_{k}^{\prime \prime}\right| \\
& \left.\times\left[Q^{(\mathrm{g})}(1) V_{i k}(13)^{\mathrm{e}} \chi_{3}^{(13)}+Q^{(\mathrm{g})}(2) V_{j k}(23)^{\mathrm{e}} \chi_{3}^{(23)}\right]\left|\lambda_{i} \lambda_{j}^{\prime} \lambda_{k}^{\prime \prime}\right\rangle_{\mathrm{A}}\right) \\
& +\left\langle\gamma_{i} \gamma_{j}^{\prime}\left|Q^{(\mathrm{g})}(1) Q^{(\mathrm{g})}(2) V_{i j} \Psi_{2}^{\mathrm{e}}\right| \lambda_{i} \lambda_{j}^{\prime}\right\rangle_{\mathrm{A}} \\
& +\left\langle\gamma_{i} \gamma_{j}^{\prime}\left|\left(\sum_{(\mathrm{g}, \mathrm{e})} Q^{(\mathrm{g})}(1) Q^{(\mathrm{e})}(2)\right) V_{i j} \Psi_{2}^{\mathrm{g}}\right| \lambda_{i} \lambda_{j}^{\prime}\right\rangle_{\mathrm{A}} \\
& +\sum_{k=1}^{2} \sum_{\lambda_{k}^{\prime \prime}}\left\langle\gamma_{i} \gamma_{j}^{\prime} \lambda_{k}^{\prime \prime}\left|T_{k}(3) S_{3}^{\mathrm{e}}\right| \lambda_{i} \lambda_{j}^{\prime} \lambda_{k}^{\prime \prime}\right\rangle_{\mathrm{A}} \\
& +\frac{1}{2} \sum_{k=1}^{2} \sum_{l=k}^{2} \sum_{\lambda_{k}^{\prime \prime} \lambda_{i}^{\prime \prime}}\left\langle\gamma_{i} \gamma_{j} \lambda_{k}^{\prime \prime} \lambda_{l}^{\prime \prime \prime}\left|\left(2-\delta_{k l}\right) V_{k l}(34)^{\mathrm{e}} \chi_{4}^{(34)}\right| \lambda_{i} \lambda_{j}^{\prime} \lambda_{k}^{\prime \prime} \lambda_{l}^{\prime \prime \prime}\right\rangle_{\mathrm{A}} \\
& \left.-\left(E_{\mathrm{e}}-E_{\mathrm{g}}\right)\left\langle\gamma_{i} \gamma_{j}^{\prime}\left|S_{2}^{\mathrm{e}}\right| \lambda_{i} \lambda_{j}^{\prime}\right\rangle_{\mathrm{A}}\right]=0
\end{aligned}
$$

where the index $i, \ldots, l$ represents particles of type $n$ when equal to $n$ and

$$
\begin{aligned}
& Q^{(\mathrm{g})} \equiv \mathbb{\nabla}-\sum_{\lambda} \Psi_{1}^{\mathrm{g}}|\lambda\rangle\left\langle\lambda\left|\quad Q^{(\mathrm{e})} \equiv-\sum_{\lambda} \Psi_{1}^{\mathrm{e}}\right| \lambda\right\rangle\langle\lambda| \\
& \left\langle\left.\gamma_{1} \gamma_{2} \gamma_{3} \gamma_{4}\right|^{\mathrm{e}} \chi_{4}^{(34)} \mid \lambda_{1} \lambda_{2} \lambda_{3} \lambda_{4}\right\rangle \\
& =\sum_{(\mathrm{g}, \mathrm{e})}\left\{S_{34}\left\langle\gamma_{3}\left|\Psi_{1}^{\mathrm{g}}\right| \lambda_{3}\right\rangle\left\langle\gamma_{1} \gamma_{2} \gamma_{4}\left|S_{3}^{\mathrm{e}}\right| \lambda_{1} \lambda_{2} \lambda_{4}\right\rangle\right. \\
& \left.+S_{12}\left\langle\gamma_{1} \gamma_{3}\left|S_{2}^{\mathrm{g}}\right| \lambda_{1} \lambda_{3}\right\rangle\left\langle\gamma_{2} \gamma_{4}\left|S_{2}^{\mathrm{e}}\right| \lambda_{2} \lambda_{4}\right\rangle\right\} \\
& +\left\langle\gamma_{1} \gamma_{2} \gamma_{3} \gamma_{4}\left|S_{4}^{\mathrm{e}}\right| \lambda_{1} \lambda_{2} \lambda_{3} \lambda_{4}\right\rangle .
\end{aligned}
$$

From equations (6.8) and (6.9) it is seen that the equation for $S_{n}^{e}$ involves $S_{n+1}^{e}$ and $S_{n+2}^{\mathrm{e}}, S_{n}^{\mathrm{g}}$ and $S_{n+1}^{\mathrm{g}}$. As in the two-component ground-state case, to use the equations in practice a truncation scheme is necessary. We employ the $\operatorname{SUB}(m, n)$ truncation and in particular the $\operatorname{SUB}(1,2)$ truncation to obtain the result linking the plasmons and poles of the relevant three-point functions.

In equations (6.8) and (6.9) there are two types of $\left\langle\left|S_{i}^{e}\right|\right\rangle$, namely $S_{1}^{e}$ for species $1-1,2-2$, and three types of $\left\langle\left|S_{2}^{\mathrm{g} / \mathrm{e}}\right|\right\rangle$, namely $S_{2}^{\mathrm{g} / \mathrm{e}}$ for species $1-1,2-2,1-2$. 


\section{RPA-like treatment of $1 \mathrm{p}-1 \mathrm{~h}$ excitations in the $\operatorname{SUB}(1,2)$ approximation}

Consider an infinite, homogeneous and overall neutral system of spin- $\frac{1}{2}$ particles of two species $i=1,2$ with masses $m_{i}$ and charges $e_{i}$. These particles interact via the following pairwise spin-independent local potentials: $V_{i i}$ between species $i-i, V_{12}$ between species 1-2. These potentials have the momentum space representation given in $\$ 3$.

We choose our single-particle basis to consist of plane-wave states with quantum labels specified by equation (3.12). We now introduce the convenient notation:

$$
\begin{aligned}
& X_{1 ; k_{1}}^{\mathrm{e} s_{1}}(\boldsymbol{q}) \equiv\left\langle\boldsymbol{k}_{1}+\boldsymbol{q}, s_{1}\left|S_{1}^{\mathrm{e}}\right| \boldsymbol{k}_{1}, s_{1}\right\rangle \\
& Y_{1 ; k_{2}}^{\mathrm{e} s_{2}}(\boldsymbol{q}) \equiv\left\langle\boldsymbol{k}_{2}+\boldsymbol{q}, s_{2}\left|S_{1}^{\mathrm{e}}\right| \boldsymbol{k}_{2}, s_{2}\right\rangle \\
& X_{2 ; \boldsymbol{k}_{1} \boldsymbol{k}_{1}^{\prime}}^{\mathrm{e}\left(\boldsymbol{q} s_{1} s_{1}^{\prime}\right.}\left(\boldsymbol{q}^{\prime}\right) \equiv\left\langle\boldsymbol{k}_{1}+\boldsymbol{q}+\boldsymbol{q}^{\prime}, s_{1} ; \boldsymbol{k}_{1}^{\prime}-\boldsymbol{q}^{\prime}, s_{1}^{\prime}\left|S_{2}^{\mathrm{e}}\right| \boldsymbol{k}_{1}, s_{1} ; \boldsymbol{k}_{1}^{\prime}, s_{1}^{\prime}\right\rangle_{\mathrm{A}} \\
& Y_{2 ; \boldsymbol{k}_{2} \boldsymbol{k}_{2}^{\prime}}^{\mathrm{e}(\boldsymbol{q}) s_{2}^{\prime} s_{2}^{\prime}}\left(\boldsymbol{q}^{\prime}\right) \equiv\left\langle\boldsymbol{k}_{2}+\boldsymbol{q}+\boldsymbol{q}^{\prime}, s_{2} ; \boldsymbol{k}_{2}^{\prime}-\boldsymbol{q}^{\prime}, s_{2}^{\prime}\left|S_{2}^{\mathrm{e}}\right| \boldsymbol{k}_{2}, s_{2} ; \boldsymbol{k}_{2}^{\prime}, s_{2}^{\prime}\right\rangle_{\mathrm{A}} \\
& Z_{2 ; \boldsymbol{k}_{1} k_{2}}^{\mathrm{e}(\boldsymbol{q}) s_{1} s_{2}}\left(\boldsymbol{q}^{\prime}\right) \equiv\left\langle\boldsymbol{k}_{1}+\boldsymbol{q}+\boldsymbol{q}^{\prime}, s_{1} ; \boldsymbol{k}_{2}-\boldsymbol{q}^{\prime}, s_{2}\left|S_{2}^{\mathrm{e}}\right| \boldsymbol{k}_{1}, s_{1} ; \boldsymbol{k}_{2}, s_{2}\right\rangle
\end{aligned}
$$

where the excited states under consideration are now eigenstates of total momentum with eigenvalue $q$. $X_{1}^{\mathrm{g}}=Y_{1}^{\mathrm{g}}=0$ by conservation of momentum and the three different types of $\left\langle\left|S_{2}^{\text {\& }}\right|\right\rangle$ are given by (3.13). Each of the three $S_{2}^{\mathrm{e}}$ can have two (generally unequal) cases: $S_{2}^{\mathrm{e} \uparrow \uparrow}=S_{2}^{\mathrm{e} \downarrow \downarrow}$ (parallel spins) and $S_{2}^{\mathrm{e} \uparrow \downarrow}=S_{2}^{\mathrm{e} \downarrow \uparrow}$ (anti-parallel spins).

Consider the $\operatorname{SUB}(1,2)$ approximation, i.e. put $S_{i}^{e}=0$ for $i>1$ and $S_{j}^{\mathrm{g}}=0$ for $j>2$ and apply it to the one-body equation (6.8). After algebraic manipulation we obtain the following equations:

$$
\begin{array}{rl}
\left(\frac{\hbar^{2}}{2 m_{i}}\left(\left|\boldsymbol{k}_{i}+\boldsymbol{q}\right|^{2}-\boldsymbol{k}_{i}^{2}\right)-\varepsilon(\boldsymbol{q})\right) S_{1 ; \boldsymbol{k}_{i}}^{e s_{i}}+T_{\mathrm{RPA}}^{(1) S}+T_{\mathrm{CHP}}^{(1) S} & \\
+T_{\mathrm{CPP}}^{(1) S}+T_{\mathrm{PH}}^{(1) S}=0 & S= \begin{cases}X & i=1 \\
Y & i=2\end{cases}
\end{array}
$$

where the superscript (1) indicates terms involving $S_{1}^{e}$, the grouping of terms is analogous to the ground-state case, and $\varepsilon(\boldsymbol{q})=E_{\mathrm{e}}(\boldsymbol{q})-E_{\mathrm{g}}$.

We only write down explicitly the $T_{\text {RPA }}$ terms. The remaining terms can be found in Lahoz (1986). In equation (7.2) we have

$$
\begin{aligned}
& T_{\mathrm{RPA}}^{(1) X}=\left(\sum_{\boldsymbol{k}_{1}^{\prime} \sigma_{1}} V_{11}(q) X_{1 ; \boldsymbol{k}_{1}^{\prime}}^{\mathrm{e} \sigma_{1}}(q) n\left(k_{1}^{\prime}\right) \bar{n}\left(\boldsymbol{k}_{1}^{\prime}+q\right)\right. \\
& \left.+\sum_{\boldsymbol{k}_{2}^{\prime} \sigma_{2}} V_{12}(q) Y_{1 ; k_{i}^{2}}^{\mathrm{e} \sigma_{2}}(\boldsymbol{q}) n\left(k_{2}^{\prime}\right) \bar{n}\left(\boldsymbol{k}_{2}^{\prime}+q\right)\right) \\
& \times\left[1+\sum_{k_{1}^{\prime \prime} \sigma_{1}} X_{2 ; k_{1} k_{1}^{\prime \prime}}^{\mathrm{gs} \sigma_{1}}(q) n\left(k_{1}^{\prime \prime}\right) \bar{n}\left(k_{1}^{\prime \prime}-q\right)\right] \\
& +\sum_{k_{i}^{\prime} \sigma_{1}} \sum_{k_{2}^{\prime} \sigma_{2}} V_{12}(q) X_{1 ; k_{1}}^{\mathrm{e} \sigma_{1}}(q) n\left(k_{1}^{\prime}\right) \bar{n}\left(k_{1}^{\prime}+q\right) Z_{2 ; k_{1} k_{i}^{\prime}}^{g^{s} \sigma_{1} \sigma_{2}}(q) n\left(k_{2}^{\prime}\right) \bar{n}\left(k_{2}^{\prime}-q\right) \\
& +\sum_{k_{2}^{\prime} \sigma_{2}} \sum_{k_{2}^{\prime \prime} \sigma_{2}^{\prime}} V_{22}(q) Y_{1 ; k_{2}^{\prime}}^{e \sigma_{2}}(q) n\left(k_{2}^{\prime}\right) \bar{n}\left(\boldsymbol{k}_{2}^{\prime}+q\right) Z_{2 ; k_{1} k_{2}^{\prime \prime}}^{g s_{1} \sigma_{2}^{\prime}}(q) n\left(k_{2}^{\prime \prime}\right) \bar{n}\left(\boldsymbol{k}_{2}^{\prime \prime}-q\right) \text {. }
\end{aligned}
$$


We can associate diagrams with equation (7.3). Using the standard convention and definitions:

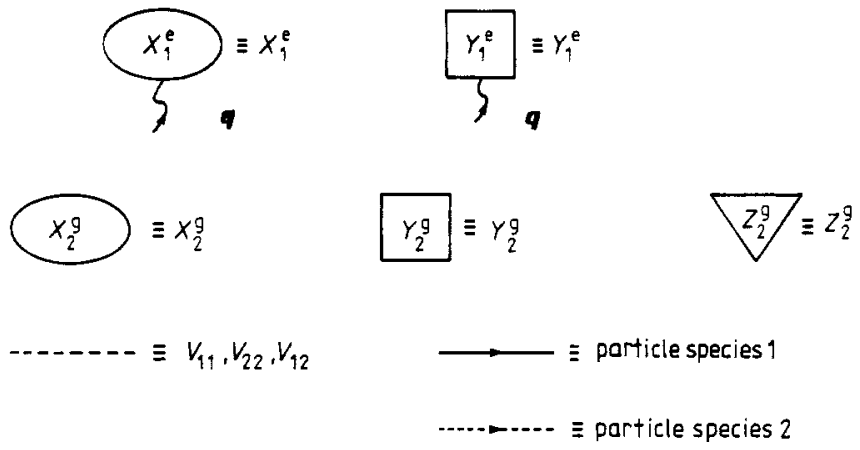

These are given by figure 17 . Figure 18 represents the diagrams associated with the other RPA term, from which the explicit equation may be derived.

Consider an RPA-like treatment of the one-body equation (7.2) where we solve:

$$
\left(\frac{\hbar^{2}}{2 m_{1}}\left(\left|k_{1}+q\right|^{2}-k_{1}^{2}\right)-\varepsilon(q)\right) X_{1 ; k_{1}}^{e s_{1}}(q)+T_{\mathrm{RPA}}^{(1) X}=0
$$
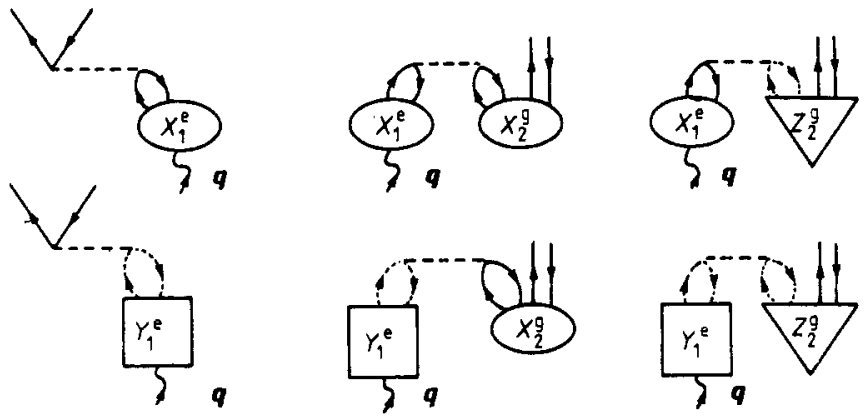

Figure 17. The diagrammatic representation of the RPA-like terms in the $\operatorname{SUB}(1,2)$ equation (7.2) for $X_{1}^{e}$, as given by equation (7.3).
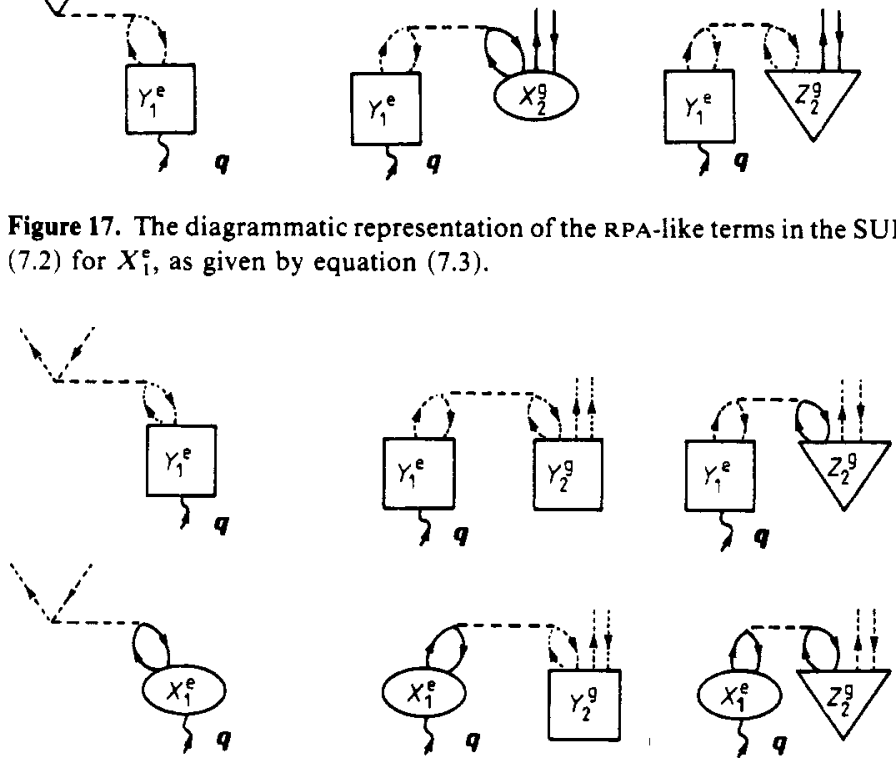

Figure 18. Same as figure 17 but for $Y_{i}^{e}$, 


$$
\left(\frac{\hbar^{2}}{2 m_{2}}\left(\left|\boldsymbol{k}_{2}+\boldsymbol{q}\right|^{2}-\boldsymbol{k}_{2}^{2}\right)-\varepsilon(\boldsymbol{q})\right) \boldsymbol{Y}_{1 ; \boldsymbol{k}_{2}}^{e s_{2}}(\boldsymbol{q})+T_{\mathrm{RPA}}^{(1) Y}=0
$$

and derive the relation between the poles of the relevant three-point functions and the plasmons.

Assume the potentials to be Coulombic and that $\left\langle\left|S_{1}^{\mathrm{e}}\right|\right\rangle,\left\langle\left|S_{2}^{\mathrm{g}}\right|\right\rangle$ are spin independent as is necessarily true in the RPA. Using the two-component results (7.4) becomes

$$
\begin{aligned}
& {\left[\boldsymbol{\kappa}_{1} \cdot \boldsymbol{q}-\tilde{\omega}(\boldsymbol{q})\right] \boldsymbol{X}_{1 ; \boldsymbol{\kappa}_{1}}^{\mathrm{e}}(\boldsymbol{q})} \\
& \qquad=\frac{-1}{2 \pi \beta} G_{1}\left(\boldsymbol{\kappa}_{1}\right)\left(\int_{\tilde{\Gamma}_{1}} \mathrm{~d} \boldsymbol{\kappa}_{1}^{\prime} X_{1 ; \boldsymbol{\kappa}_{1}}^{\mathrm{e}}(\boldsymbol{q})-\int_{\tilde{\Gamma}_{2}} \mathrm{~d} \boldsymbol{\kappa}_{2}^{\prime} Y_{1 ; \boldsymbol{\kappa}_{2}^{\prime}}^{\mathrm{e}}(\boldsymbol{q})\right)
\end{aligned}
$$

where we have made the momenta dimensionless, changed the sum to an integral in the standard manner, assumed $k_{\mathrm{F}_{1}}=k_{\mathrm{F}_{2}}=k_{\mathrm{F}}$, introduced the more symmetric momentum variables $\boldsymbol{\kappa}_{i}$ by the relation $\boldsymbol{k}_{i}=\boldsymbol{\kappa}_{i}-\frac{1}{2} \boldsymbol{q}$ as before and defined $\beta$ and $\tilde{\Gamma}_{i}$ as in $\S 4$ and

$$
\tilde{\omega}(\boldsymbol{q}) \equiv\left(m_{1} / \hbar^{2}\right) \varepsilon(\boldsymbol{q})
$$

Changing to cylindrical polar coordinates in the usual manner (Lahoz 1986) yields

$$
X_{1}^{\mathrm{e}}\left(x_{1}\right)=\frac{-G_{1}\left(x_{1}\right)\left[I^{(1)}-I^{(2)}\right]}{\beta\left(x_{1}-\tilde{\omega}(\boldsymbol{q}) / q\right)}
$$

where we have defined $x_{i} \equiv \kappa_{i} \cdot \hat{q}$ and the integrals

$$
\begin{aligned}
I^{(1)} & \equiv \int_{\tilde{L}_{1}} \mathrm{~d} x_{1}^{\prime}\left[\tilde{N}_{1}\left(x_{1}^{\prime}\right)+M \tilde{N}_{2}\left(M x_{1}^{\prime}\right)\right] X_{1}^{\mathrm{s}}\left(x_{1}^{\prime}\right) \\
I^{(2)} & \equiv \int_{M \tilde{L}_{2}} \mathrm{~d} x_{2}^{\prime}\left[\tilde{N}_{2}\left(x_{2}^{\prime}\right)+\tilde{N}_{1}\left(x_{2}^{\prime} / M\right) / M\right] Y_{1}^{\mathrm{e}}\left(x_{2}^{\prime}\right)
\end{aligned}
$$

where $M^{i-1} \tilde{L}_{i}, \tilde{N}_{i}\left(x_{\mathrm{i}}\right)(i=1,2)$ are well known from the ground-state results. Straightforward manipulation of equation (7.7) and its particle- 2 counterpart and use of the ground-state results yields:

$$
K(\tilde{\omega}(q) / q) G_{1}(\tilde{\omega}(q) / q)=0
$$

which, on further use of the functional relation (4.22), becomes

$$
\frac{1}{G_{1}(-\tilde{\omega}(q) / q)}=0
$$

which shows that the pole of $G_{1}\left(z_{1}\right)$ is the negative of the zero, i.e. plasmon of the dielectric function $K\left(z_{1}\right)$. Similar considerations yield the analogous result linking the pole of $H_{1}\left(z_{2}\right)$ to the plasmon of the dielectric function $L\left(z_{2}\right)$.

The non-Coulombic case analogue of the above result is derived in a similar manner (Lahoz 1986). The one-component result, which can be derived from our results, was originally obtained by Bishop and Lührmann (1981). Clearly, the CCM yields the result (analogous to that in ordinary two-body potential scattering) which links the cut of the three-point function to the particle-hole scattering continuum and the pole to a bound state. Further, we see that the bound state is not a two-body bound state but a true (many particle-hole) collective excitation of the many-body system.

The full two-component SUB $(2,2)$ equations can be found in Lahoz (1986). Somewhat surprisingly perhaps, the RPA-like $\operatorname{SUB}(2,2)$ equations appear not to be susceptible 
to analytic solution, just as in the comparable one-component case. Evidently, numerical work is required to proceed further with such a calculation. In contrast, the complete ladder-like (CLAD like) subapproximation to the $\operatorname{SUB}(2,2)$ equations is expected to possess an analytic solution in the two-component case, as for the one-component case investigated by Stevens (1984).

\section{Conclusions and further work}

In this first paper of two, we generalise the one-component $\mathrm{CCM}$ results in the fluid ground-state and fluid excited-state formalisms to two components with particular emphasis on homogeneous fermion systems. In the second paper we generalise the one-component CCM results in the BCS ground-state formalism to two components. The motivation for this work is that systems such as real metals and electron-hole plasmas in photoexcited semiconductors, and phenomena such as superconductivity, can be described in terms of two-component systems. The outstanding success of the one-component СCM naturally leads us to regard its two-component version as the best way to handle them.

Consequently, in this paper we develop the two-component formalism for the fluid ground and excited states for Coulombic $\left(V_{11} V_{22}=V_{12}^{2}\right)$ and non-Coulombic $\left(V_{11} V_{22} \neq\right.$ $V_{12}^{2}$ ) potentials, and consider the well known random-phase approximation (RPA), which describes the high-density and long-range limit, in the ground-state and the RPA-like approximation in the excited states.

Our RPA treatment of $2 \mathrm{p}-2 \mathrm{~h}$ correlations using the SUB2 approximation yields analytic solutions for a general finite mass ratio $M$ and an expression for the correlation energy in the Coulombic case. The cases $M=1$, providing a first approximation to an electron-hole system, and $M=1836$, providing a description of a hydrogen plasma, are investigated and found to be in excellent qualitative agreement with results from variational methods. The Born-Oppenheimer approximation $(\lim M \rightarrow \infty)$ is implemented and the correct physical result obtained. In the non-Coulombic case we indicate how to obtain a (possible) unique solution. We note in passing that our RPA analysis also provides a technique for solving certain kinds of non-linear integral equations by means of Muskhelishvili-Omnès theory.

The RPA-like treatment of $1 \mathrm{p}-1 \mathrm{~h}$ excitations using the $\operatorname{SUB}(1,2)$ approximation yields the result linking the cut of the three-point functions introduced in the solution of the ground-state RPA equations to the particle-hole scattering continuum, and the pole of the same three-point functions to a bound state. This result, analogous to that obtained in ordinary two-body potential scattering theory, serves to reinforce the suitability of the CCM for dealing with many-body systems.

We feel that a full SUB2 treatment of the two-component ground-state equation, using the RPA Coulombic result to derive insight into how best to perform such an extension and check the necessary numerical approximations, will prove just as successful as in the one-component case. Similar considerations (cf Stevens 1984) indicate the suitability of performing a complete ladder (CLAD) treatment of the SUB2 equations, describing the low-density and short-range limit, to obtain an analytic solution. We also feel that the RPA non-Coulombic potential equations obtained will provide a first approximation to a real metal consisting of mobile electrons with a background of relatively immobile ions, the ion-ion and electron-electron interactions being Coulombic in nature and the electron-ion interaction obtained from solid state theory. A full 
SUB2 non-Coulombic treatment, analogous to that in the Coulombic case, ought to be attempted.

We believe that numerical work in the excited-state formalism concerning an RPA-like treatment of $2 \mathrm{p}-2 \mathrm{~h}$ excitations in the $\operatorname{SUB}(2,2)$ approximation should also be implemented. A complete ladder (CLAD)-like treatment of $2 \mathrm{p}-2 \mathrm{~h}$ excitations in the $\operatorname{SUB}(2,2)$ approximation, analogous to that in the one-component case (cf Stevens 1984), ought to be attempted in order to obtain an analytic solution and the twocomponent analogue of the 'de-excited states', i.e. states with negative excitation energy and, consequently, lower energy than the so-called ground state. These de-excited states' are intimately connected with phase transitions and the question of what is the best choice of model state for solving the Schrödinger equation. A full discussion of these points is deferred until we discuss superconductivity in paper II, where by studying the necessary conditions for superconductivity in our two-component formalism, we find we are able to compare the different model states investigated and shed light on the problem of phase transitions and what particular approximations yield these.

\section{Acknowledgment}

RFB gratefully acknowledges support for this work in the form of a research grant from the Science and Engineering Research Council of Great Britain.

\section{References}

Arponen J 1983 Ann. Phys., NY 151311

Bardeen J, Cooper L N and Schrieffer J R 1957 Phys. Rev. 1081175

Bartlett R J and Purvis G D 1980 Phys. Scr. 21255

Bethe H A and Goldstone J 1957 Proc. R. Soc. A 238551

Bishop R F 1985 An. Fis. A 819

Bishop R F and Kümmel H 1987 Phys. Today 40(3) 52

Bishop R F and Lahoz W A 1987 in preparation

Bishop R F and Lührmann K H 1978 Phys. Rev. B 173757

- 1981 unpublished

1982 Phys. Rev. B 265523

Bogoliubov N N 1958 Zh. Eksp. Teor. Fiz. 3458 (Sov. Phys.-JETP 741 )

Bohm D and Pines D 1953 Phys. Rev. 92609

Born M and Oppenheimer J R 1927 Ann. Phys. Lpz. 84457

Ceperley D M and Alder B J 1980 Phys. Rev. Lett. 45566

Chakraborty T, Kallio A, Lantto L J and Pietiläinen P 1983 Phys. Rev. B 273061

Chakraborty T and Pietiläinen P 1982 Phys. Rev. Lett. 491034

Chisholm J S R and Squires E J 1959 Nucl. Phys. 13156

Čižek J 1966 J. Chem. Phys. 454256

1969 Adv. Chem. Phys. 1435

Coester F 1958 Nucl. Phys. 7421

1969 Lectures in Theoretical Physics vol 11, ed Mahanthappa K H (New York: Gordon and Breach) Coester F and Kümmel H 1960 Nucl. Phys. 17477

Cooper L N 1956 Phys. Rev. 1041189

Dancoff S M 1950 Phys. Rev. 78382

Emrich K 1981a Nucl. Phys. A 351379

- 1981b Nucl. Phys. A 351397

Emrich K and Zabolitzky J G 1984 Phys. Rev. B 302049

1985 Preprint Bochum 
Fetter A L and Walecka J D 1971 Quantum Theory of Many-Particle Systems (New York: McGraw-Hill) Gell-Mann M and Brueckner K 1957 Phys. Rev. 106364

Haque A and Kaldor U 1987 Int. J. Quantum. Chem. Symp. to be published

Hubbard J 1957 Proc. R. Soc. A 243336

Knox R S 1963 Theory of Excitons (New York: Academic)

Kümmel H 1984 Nucleon-Nucleon Interaction and Nuclear Many-Body problems ed S S Wu and T T S Kuo (Singapore: World Scientific)

Kümmel H, Lührmann K H and Zabolitzky J G 1978 Phys. Rep. C. 361

Kvasnička V, Laurinc V and Biskupič S 1982 Phys. Rep. C 90160

Lahoz W A 1986 PhD Thesis Manchester University

Lindgren I 1978 Int. J. Quantum. Chem. Symp. 1233

Lipkin H J, Meshkov N and Glick J 1965a Nucl. Phys. 62188

1965b Nucl. Phys. 62199

1965c Nucl. Phys. 62211

Lührmann K H 1977 Ann. Phys., NY 103253

Macke W 1950 Z. Naturf. A 5192

Mehta M L 1959 Nucl. Phys. 12333

Muskhelishvili N I 1953 Singular Integral Equations (Groningen: Noordhoff)

Nesbet R K 1958 Phys. Rev. 1091632

Offermann R 1975 PhD Thesis Ruhr Universität Bochum

1976 Nucl. Phys. A 273368

Offermann R, Kümmel H and Ey W 1976 Nucl. Phys. A 273349

Omnès R 1958 Nuovo Cimento 8316

Primas H 1965 Modern Quantum Chemistry vol II, ed O Sinanoglu (New York: Academic) p 45

Robinson N I 1986 PhD Thesis Manchester University

Schiff L I 1968 Quantum Mechanics (New York: McGraw-Hill) 3rd edn

Stevens G A 1984 PhD Thesis Manchester University

Szalewicz K, Zabolitzky J G, Jeziorski B and Monkhorst H J 1984 J. Chem. Phys. 812723

Tamm 11945 J. Phys. (USSR) 9449

Valatin J 1958 Nuovo Cimento 7843

Wigner E 1934 Phys. Rev. 461002

_- 1938 Trans. Faraday Soc. 34678

Zabolitzky J G 1974a Nucl. Phys. A 228272

— 1974b Nucl. Phys. A 228285 